Article

\title{
A New Nine-Dimensional Chaotic Lorenz System with Quaternion Variables: Complicated Dynamics, Electronic Circuit Design, Anti-Anticipating Synchronization, and Chaotic Masking Communication Application
}

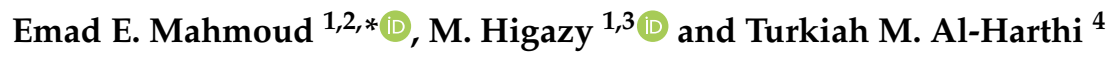 \\ 1 Department of Mathematics, Faculty of Science, Taif University, Taif 888, Saudi Arabia; M.Higazy@tu.edu.sa \\ 2 Department of Mathematics, Faculty of Science, Sohag University, Sohag 82524, Egypt \\ 3 Department of Physics and Engineering Mathematics, Faculty of Electronic Engineering, Menoufia \\ University, Menouf 32952, Egypt \\ 4 Department of Mathematics, Shaqra University, Shaqra 11921, Saudi Arabia; talharthy@su.edu.sa \\ * Correspondence: e.mahmoud@tu.edu.sa; Tel.: +966508733478
}

Received: 30 July 2019; Accepted: 18 September 2019; Published: 20 September 2019

\begin{abstract}
In this paper, a chaotic quaternion autonomous nonlinear structure is introduced and intends to be a contribution. It is the first nonlinear dynamical system with quaternion variables to be studied in the literature. With nine dimensions, the new system is a high-dimensional one. Several vital characteristics and features of this model are investigated, such as its Hamiltonian, symmetry, signal flow graph, dissipation, equilibriums and their stability, Lyapunov exponents, Lyapunov dimension, bifurcation diagrams, and chaotic behavior. A circuit implementation is designed to realize the new system, and a scheme is designed to achieve anti-anticipating synchronization (AAS) of two identical chaotic attractors with quaternion variables based on a Lyapunov function and active control. The concept of AAS is yet to be explored in the literature. A simulation experiment is designed and executed to illustrate the effectiveness of the acquired results. After synchronization, numerical outcomes are planned to explain the status variables and errors of these chaotic attractors to prove that AAS is achieved. The secure communication problem is studied based on the obtained events of the AAS of two identical nonlinear Lorenz systems with quaternion variables. AAS connecting the drive and response systems in chaotic systems with quaternion variables is the key to achieving communication. Signal encryption and restoration are simulated numerically.
\end{abstract}

Keywords: quaternion; chaotic; lyapunov function; anti-anticipating synchronization; secure communication

\section{Introduction}

In 1843 [1], William Hamilton first reported the concept of quaternion numbers and used them to study basic mechanics in three-dimensional space. The most critical feature of quaternions is that multiplying two quaternions is noncommutative. Quaternions have applications in graphics, flight dynamics, orbital mechanics of satellites, crystallographic texture analysis, and especially communication [2]. Chaotic systems based on quaternion variables have advantages over those based on real or complex variables in that the former provide secure communication because of (i) the increased number of dimensions needed to intercept communication and (ii) the feasibility of being implemented using capacitors and inductors. It is found that the real and imaginary parts of quaternion variables lead to complicated computation, thereby quadrupling the transmitted 
contents and improving the security of communication. Moreover, quaternion variables are more easily implemented by RLC circuits comprising resistors (R), inductors (L), and capacitors (C), thereby extending the practical applications of quaternion-variable chaotic systems.

Chaotic synchronization in physical, chemical, and biological systems has become an attractive subject in recent years and has a distinctly modern focus [3], with remarkable applications in secure communication. A secure communication system requires the development of a signal with the data that interceptors inside a carrier or transmitter signal must continue to remain undetectable. Such data or information can be secured by placing them or it in a chaotic signal that is transmitted to a recommended receiver that can detect and recover the information from the chaotic signal. The following lines show that there exist many studies that help to build these applications.

The work of Voss $[4,5]$ has led to the proven possibility of synchronizing autonomous dynamical systems in a drive-response configuration such that the response system can actually anticipate (i.e., predict) the trajectory of the drive system. This remarkable phenomenon is achieved by introducing appropriate dynamics of the response system. Since then, there have been some reports on anticipating synchronization [6-8], which has many applications in semiconductor lasers, chaotic laser diodes, and cryptography. Herein, we consider what happens if the response system expected behavior of the drive system with opposite shape. We deal with a new type of synchronization that we refer to as anti-anticipating synchronization (AAS). To the best of our knowledge, AAS is yet to be studied in the literature.

Chaos is an umbrella term for various complex behaviors of solutions to relatively simple deterministic systems. Because chaos theory has various important applications in a wide range of fields (e.g., studying the dynamics of biological signals, many types of data and image encryption, investigating sunspot cycles [9], and laser applications [10]), it has attracted much interest in studying the complex behaviors of nonlinear systems. The study of chaos came into its own in 1963 with the work of Edward Lorenz. Lorenz was developing a model of convection in the atmosphere, and he found a remarkable property whereby solutions that passed through nearby points would diverge from each other extremely rapidly [11] but also remain confined within a certain space. Specifically, the Lorenz attractor consists of a set of chaotic solutions of the Lorenz system that, when plotted, look like a butterfly or a figure of eight. This was later found to be characteristic of many other systems, thus beginning the study of chaotic dynamics [12-14]. Chaotic models have one positive Lyapunov exponent, and the following set of differential equations describes this chaotic Lorenz model:

$$
\begin{aligned}
\dot{x}(t) & =\alpha(y(t)-x(t)) \\
\dot{y}(t) & =\gamma x(t)-y(t)-x(t) z(t) \\
\dot{z}(t) & =-\beta z(t)+x(t) y(t) .
\end{aligned}
$$

For $\alpha=10, \beta=\frac{8}{3}$, and $\gamma=28$, this system has only one two-scroll chaotic attractor. The literature has several studies of this system in the fields related to the chaos theory, dynamical systems, synchronization and control of chaotic systems. Subsequently, several algorithms for analyzing and synthesizing chaotic (or hyperchaotic) systems of real variables have been proposed [15-17].

In 1982, Fowler et al. generalized the real Lorenz system by introducing the complex Lorenz equations [18-20]:

$$
\begin{aligned}
\dot{x}(t) & =\alpha(y(t)-x(t)) \\
\dot{y}(t) & =\gamma x(t)-y(t)-x(t) z(t) \\
\dot{z}(t) & =-\beta z(t)+\frac{1}{2}(y(t) \bar{x}(t)+x(t) \bar{y}(t))
\end{aligned}
$$

where the attidute of the system is controlled via the positive control parameters $\alpha, \beta$, and $\gamma, x(t)=$ $v_{1}(t)+i v_{2}(t)$ and $y(t)=v_{3}(t)+i v_{4}(t)$ are complex variables, and $z(t)=v_{5}(t)$ is a real variable. A dot symbolizes the derivative with respect to time, an overbar symbolizes the complex conjugate, 
and $i^{2}=-1$. The chaotic complex Lorenz system (2) is therefore described by a five-dimensional (5D) system of real first-order autonomous ordinary differential equations. The physics of detuned lasers and the thermal convection of liquid flows are described and simulated via the complex Lorenz equations [21], where the complex variables are used to represent the electric field and the atomic polarization amplitude.In addition, one interesting possibility of the study of complex systems is that of modelling biological systems such as humans. For example, nonlinear systems have been used to characterize the human (see for example [22] and the references cited there).

Mahmoud et al. have proposed several chaotic and hyperchaotic complex systems [23,24], and such systems are known to have much-wider applications. For example, chaotic and hyperchaotic complex systems are useful when studying the amplitudes of electromagnetic fields and the atomic polarization of laser systems. They can also be used for communication, where doubling the number of variables can help increase the content and security of the transmitted information $[25,26]$. In the present work, we focus on chaotic systems with quaternion variables because of their importance in securing the transfer of information. The main aim of the present paper is to introduce the first chaotic nonlinear dynamical system with hypercomplex (quaternion) variables in the form

$$
\begin{aligned}
& \dot{x}(t)=\alpha(y(t)-x(t)) \\
& \dot{y}(t)=\gamma x(t)-y(t)-x(t) z(t), \\
& \dot{z}(t)=-\beta z(t)+\frac{1}{2}(y(t) \bar{x}(t)+x(t) \bar{y}(t)),
\end{aligned}
$$

where $x(t)=v_{1}(t)+i v_{2}(t)+j v_{3}(t)+k v_{4}(t), y(t)=v_{5}(t)+i v_{6}(t)+j v_{7}(t)+k v_{8}(t), z(t)=v_{9}(t), i^{2}=$ $j^{2}=k^{2}=i j k=-1$, and an overbar symbolizes the quaternion conjugate. The proposed new system (3) is a generalization of the complex and real Lorenz systems and is referred to as a hypercomplex Lorenz system. This new model is a nine-dimensional (9D) system of real first-order autonomous ordinary differential equations. Herein, we study its dynamics, basic properties, AAS, and application to secure communication.

Note that this new system is limited to quadratic nonlinearity, particularly $x z, y \bar{x}$, and $x \bar{y}$. For the system to retain its identity, we must maintain this multiplicative order because the quaternion group is non-commutative.

This paper is organized as follows. In Section 2, the basics of quaternion numbers are reviewed. In Section 3, the essential characteristics of system (3) are studied, namely its Hamiltonian, symmetry, signal flow graph, invariance, and fixed points with stability analysis of the trivial and nontrivial fixed points. The quaternion behavior of system (3) is investigated. In the light of the computations of Lyapunov exponents, the range of parameter values of the model in which chaotic attractors exist is computed numerically, and the dynamics of (3) are classified well based on the indications of the Lyapunov exponents. The Lyapunov dimension is investigated and determined for the chaotic attractors of (3). We use bifurcation analysis to characterize and distinguish the chaotic behavior of (3). In Section 4, a circuit implementation and Simulink simulation are constructed. In Section 5, a definition of AAS is introduced, a new scheme is designed to achieve AAS based on a Lyapunov function, and the results of the AAS of (3) are used to construct a simple application to secure communication. Finally, conclusions are drawn in Section 6.

\section{Basics of Quaternion Numbers}

Mathematically, the quaternion number system is a continuation of complex numbers. Quaternions were first presented in 1843 [27] by the Irish mathematician William Rowan Hamilton as a means of reviewing basic mechanics in three-dimensional space. One prominant example of quaternions is that of quantum mechanics (see [28]). An example of the characteristics of quaternions is that multiplication of two quaternions is noncommutative [28]. As in [28], a quaternion is characterized as a two-line quotient in a three-dimensional space or as a two-vector 
quotient as in [29]. Quaternions have several applications and are used in both pure and applied mathematics, particularly for applications requiring three-dimensional rotations in computer graphics, computer vision [30], and spectrographic texture analysis. They can also be used in practical applications alongside or as an option to other methods, such as Euler angles and rotation matrices [31]. Advanced physics deals with some quaternion roles [32]. It explicates how different physical covariance groups can be related to the quaternion group: the Lorentz group, the general relativity group, and the conformal group [33,34]. A general quaternion number is written as $q=a+b i+c j+d k$, where $a, b, c$, and $d$ are real numbers and $i, j$, and $k$ are the fundamental quaternion units. For more details about the basics of quaternion numbers, see Refs. [27,28].

\section{Properties of Dynamical Behavior of Model (3)}

In this section, some characteristics of the model (3) are investigated, for which the elements of the chaotic system are extracted using Lyapunov exponents and bifurcation diagrams. Separating the real and imaginary parts of (3) leads to

$$
\begin{aligned}
& \dot{v}_{1}(t)=\alpha\left(v_{5(t)}-v_{1(t)}\right) \\
& \dot{v}_{2}(t)=\alpha\left(v_{6(t)}-v_{2(t)}\right) \\
& \dot{v}_{3}(t)=\alpha\left(v_{7(t)}-v_{3(t)}\right) \\
& \dot{v}_{4}(t)=\alpha\left(v_{8(t)}-v_{4(t)}\right) \\
& \dot{v}_{5}(t)=\gamma v_{1(t)}-v_{5(t)}-v_{1(t)} v_{9(t)}, \\
& \dot{v}_{6}(t)=\gamma v_{2(t)}-v_{6(t)}-v_{2(t)} v_{9(t)} \\
& \dot{v}_{7}(t)=\gamma v_{3(t)}-v_{7(t)}-v_{3(t)} v_{9(t)} \\
& \dot{v}_{8}(t)=\gamma v_{4(t)}-v_{8(t)}-v_{4(t)} v_{9(t)}, \\
& \dot{v}_{9}(t)=-\beta v_{9(t)}+v_{1(t)} v_{5(t)}+v_{2(t)} v_{6(t)}+v_{3(t)} v_{7(t)}+v_{4(t)} v_{8(t)} .
\end{aligned}
$$

The basic dynamical characteristics of (4) are as follows.

\subsection{Generalization of Hamiltonian for (4)}

We consider $\mathbf{x}=\left[v_{1}(t), v_{2}(t), v_{3}(t), v_{4}(t), v_{5}(t), v_{6}(t), v_{7}(t), v_{8}(t), v_{9}(t)\right]^{T}, \mathbf{H}(\mathbf{x})$ is the globally positive-definite Hamiltonian continuous energy function and its gradient vector is $\frac{\partial \mathbf{H}}{\partial \mathbf{x}}$.

Then, we can rewrite the continuous nonlinear model (4) in the following form [35]:

$$
\dot{\mathbf{x}}=(\boldsymbol{\tau}(\mathbf{x})+\sigma(\mathbf{x})) \frac{\partial \mathbf{H}}{\partial \mathbf{x}} .
$$

By using quadratic energy [35]:

$$
\mathbf{H}(\mathbf{x})=\frac{1}{2} \mathbf{x}^{T} \gamma \mathbf{x}
$$

where $\gamma$ is a symmetric and positive-definite constant diagonal matrix, with regard to $\frac{\partial \mathbf{H}}{\partial \mathbf{x}}=\gamma \mathbf{x}$ [35], the workless part of the vector field is represented by the antisymmetric matrix $\boldsymbol{\tau}(\mathbf{x})$ and the working or nonconservative part of the model is represented by the negative-definite symmetric matrix $\sigma(\mathbf{x})$ [35]. That is,

$$
\boldsymbol{\tau}(\mathbf{x})=[-\boldsymbol{\tau}(\mathbf{x})]^{T} \text { and } \sigma(\mathbf{x})=[\sigma(\mathbf{x})]^{T} .
$$

Now, $\mathbf{H}(\mathbf{x})$ of (4) can be defined as

$$
\mathbf{H}(\mathbf{x})=\frac{1}{2}\left[\frac{\left(v_{1}\right)^{2}}{\alpha}+\frac{\left(v_{2}\right)^{2}}{\alpha}+\frac{\left(v_{3}\right)^{2}}{\alpha}+\frac{\left(v_{4}\right)^{2}}{\alpha}+\frac{\left(v_{5}\right)^{2}}{\gamma}+\frac{\left(v_{6}\right)^{2}}{\gamma}+\frac{\left(v_{7}\right)^{2}}{\gamma}+\frac{\left(v_{8}\right)^{2}}{\gamma}+\frac{\left(v_{9}\right)^{2}}{\gamma}\right] .
$$


In this way, system (4) can be acquired based on Equation (5) as:

$$
\begin{aligned}
{\left[\begin{array}{c}
\dot{v}_{2}(t) \\
\dot{v}_{2}(t) \\
\dot{v}_{3}(t) \\
\dot{v}_{4}(t) \\
\dot{v}_{5}(t) \\
\dot{v}_{6}(t) \\
\dot{v}_{7}(t) \\
\dot{v}_{8}(t) \\
\dot{v}_{9}(t)
\end{array}\right]=\left[\begin{array}{ccccccccc}
0 & 0 & 0 & 0 & 0 & 0 & 0 & 0 & 0 \\
0 & 0 & 0 & 0 & 0 & 0 & 0 & 0 & 0 \\
0 & 0 & 0 & 0 & 0 & 0 & 0 & 0 & 0 \\
0 & 0 & 0 & 0 & 0 & 0 & 0 & 0 & 0 \\
0 & 0 & 0 & 0 & 0 & 0 & 0 & 0 & -\gamma v_{1} \\
0 & 0 & 0 & 0 & 0 & 0 & 0 & 0 & -\gamma v_{2} \\
0 & 0 & 0 & 0 & 0 & 0 & 0 & 0 & -\gamma v_{3} \\
0 & 0 & 0 & 0 & 0 & 0 & 0 & 0 & -\gamma v_{4} \\
0 & 0 & 0 & 0 & \gamma v_{1} & \gamma v_{2} & \gamma v_{3} & \gamma v_{4} & 0
\end{array}\right]\left[\begin{array}{c}
\frac{v_{1}}{\alpha} \\
\frac{v_{2}}{\alpha} \\
\frac{v_{3}}{\alpha} \\
\frac{v_{4}}{\alpha} \\
\frac{v_{5}}{\gamma} \\
\frac{v_{6}}{\gamma} \\
\frac{v_{7}}{\gamma} \\
\frac{v_{8}}{\gamma} \\
\frac{v_{9}}{\gamma}
\end{array}\right] } \\
+\left[\begin{array}{ccccccccc}
2 \\
-\alpha & 0 & 0 & 0 & \alpha \gamma & 0 & 0 & 0 & 0 \\
0 & -\alpha & 0 & 0 & 0 & \alpha \gamma & 0 & 0 & 0 \\
0 & 0 & -\alpha^{2} & 0 & 0 & 0 & \alpha \gamma & 0 & 0 \\
0 & 0 & 0 & -\alpha^{2} & 0 & 0 & 0 & \alpha \gamma & 0 \\
\alpha \gamma & 0 & 0 & 0 & -\gamma & 0 & 0 & 0 & 0 \\
0 & \alpha \gamma & 0 & 0 & 0 & -\gamma & 0 & 0 & 0 \\
0 & 0 & \alpha \gamma & 0 & 0 & 0 & -\gamma & 0 & 0 \\
0 & 0 & 0 & \alpha \gamma & 0 & 0 & 0 & -\gamma & 0 \\
0 & 0 & 0 & 0 & 0 & 0 & 0 & 0 & -\gamma \beta
\end{array}\right]\left[\begin{array}{c}
\frac{v_{1}}{\alpha} \\
\frac{v_{2}}{\alpha} \\
\frac{v_{3}}{\alpha} \\
\frac{v_{4}}{\alpha} \\
\frac{v_{5}}{\gamma} \\
\frac{v_{6}}{\gamma} \\
\frac{v_{7}}{\gamma} \\
\frac{v_{8}}{\gamma} \\
\frac{v_{9}}{\gamma}
\end{array}\right] .
\end{aligned}
$$

Because model (4) has a Hamiltonian energy function, it can be used to measure physical quantities.

\subsection{Invariance and Symmetry of (4)}

(i) Assume that (4) is invariant under the transformation $\left(v_{1}(t), v_{2}(t), v_{3}(t), v_{4}(t)\right.$, $\left.v_{5}(t), v_{6}(t), v_{7}(t), v_{8}(t), v_{9}(t)\right) \Rightarrow\left(-v_{1}(t),-v_{2}(t),-v_{3}(t),-v_{4}(t),-v_{5}(t),-v_{6}(t),-v_{7}(t)\right.$, $\left.-v_{8}(t), v_{9}(t)\right)$. If $(4)$ has the clarification $\left(v_{1}(t), v_{2}(t), v_{3}(t), v_{4}(t), v_{5}(t), v_{6}(t), v_{7}(t), v_{8}(t)\right.$, $\left.v_{9}(t)\right)$, then the solution of the comparable model is defined as $\left(-v_{1}(t),-v_{2}(t),-v_{3}(t),-v_{4}(t)\right.$, $\left.-v_{5}(t),-v_{6}(t),-v_{7}(t),-v_{8}(t), v_{9}(t)\right)$.

(ii) Model (4) preserves symmetry because it is invariant if subjected to the transformation

$$
\begin{aligned}
& v_{1}=v_{1} \cos \theta-v_{2} \sin \theta, v_{2}=v_{1} \sin \theta+v_{2} \cos \theta, \\
& v_{3}=v_{3} \cos \theta-v_{4} \sin \theta, v_{4}=v_{3} \sin \theta+v_{4} \cos \theta, \\
& v_{5}=v_{5} \cos \theta-v_{6} \sin \theta, v_{6}=v_{5} \sin \theta+v_{6} \cos \theta, \\
& v_{7}=v_{7} \cos \theta-v_{8} \sin \theta, v_{8}=v_{7} \sin \theta+v_{8} \cos \theta, \\
& v_{9}=v_{9} .
\end{aligned}
$$

This shows that there is rotational symmetry about the $v_{9}$ axis of (4). Therefore, if (4) has a solution of the form $\left(v_{1}(t), v_{2}(t), v_{3}(t), v_{4}(t), v_{5}(t), v_{6}(t), v_{7}(t), v_{8}(t), v_{9}(t)\right)$, then there exists a supplementary solution of the form $\left(v_{1}(t), v_{2}(t), v_{3}(t), v_{4}(t), v_{5}(t), v_{6}(t), v_{7}(t), v_{8}(t), v_{9}(t)\right)$.

\subsection{Signal Flow Graph of (4)}

In this subsection, we produce a signal flow graph (SFG) of (4) to show the composition of the system, the interconnections between its states, and the direct impact of each state variable on the other state variables. For more details about SFGs, see, for example [36], and the references therein. As shown in Figure 1, each state variable is represented by a vertex, and there exists a directed edge between two different state variables if one has a direct effect on the other [see (4)]. The SFG of the studied system can be decomposed into four isomorphic sub-digraphs as shown in Figure 2. We refer 
to each of these subgraphs as a system generator $G_{l}$, where $1 \leq l \leq 4$ and $G=\bigcup_{l=1}^{4} G_{l}$. By careful inspection of the SFG, it is clear that all generators have a common vertex $v_{9}$. We then propose the following notation.

Notation: The studied system can be generalized using any number of generators with the general SFG $G^{*}=\bigcup_{l=1}^{n} G_{l}$ with $2 n+1$ vertices (state variables).

The above notation agrees with the mathematical generalization considered in Section 3.2.

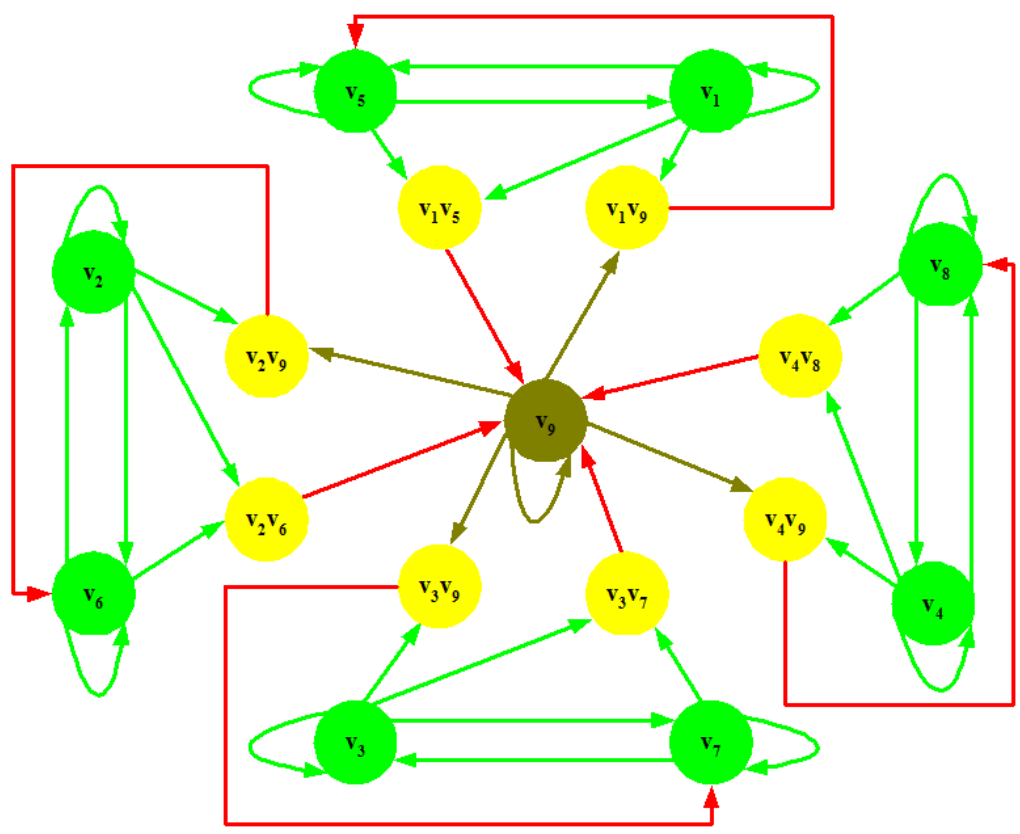

Figure 1. The signal flow graph of the system (4).

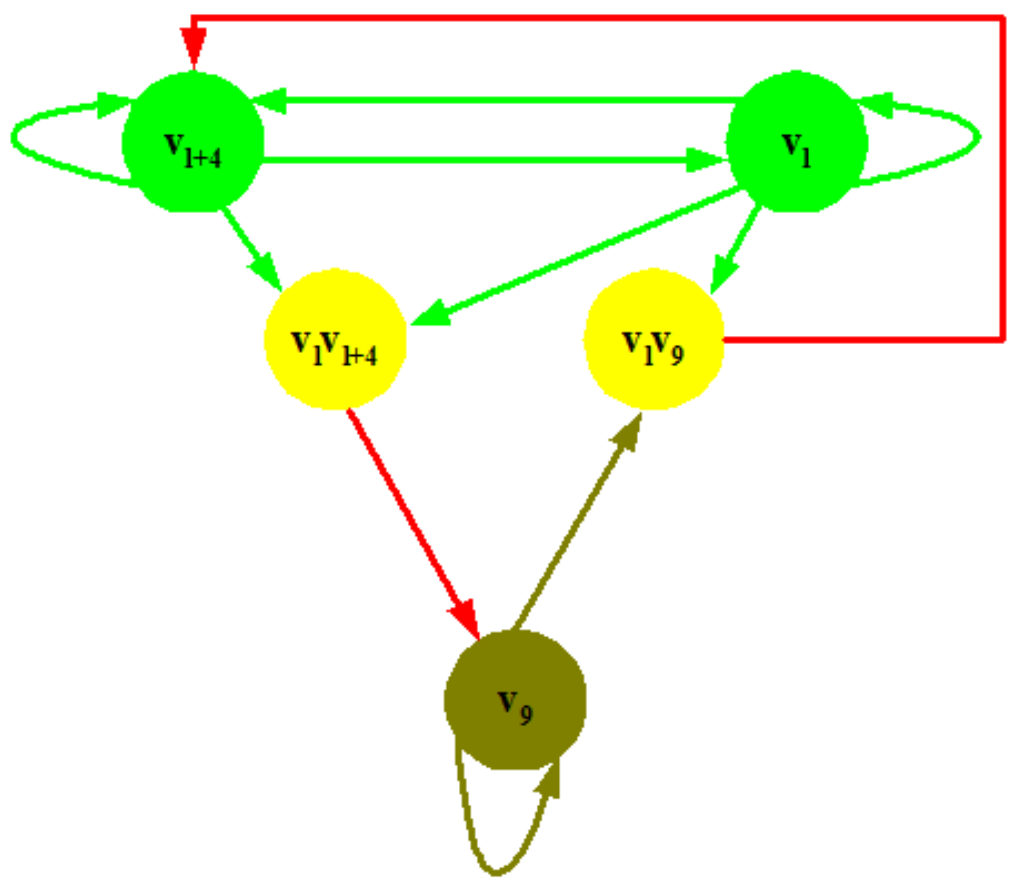

Figure 2. The generator graph of the system (4) where $1 \leq l \leq 4$. 


\subsection{Dissipation}

Model (4) can be written in vector notation as follows:

$$
\dot{\mathbf{x}}=\mathbf{f}(\mathbf{x})=\left\{\begin{array}{l}
\alpha\left(v_{h+4}-v_{h}\right), \\
\gamma v_{h}-v_{h+4}-v_{h} v_{9}, \\
-\beta v_{9}+\prod_{h=1}^{4} v_{h} v_{h+4}
\end{array}\right.
$$

where $1 \leq h \leq 4$. Assume that $\Xi(t)$ is an arbitrary region in $R^{9}$ that has a smooth boundary and assume that $\Xi(t) \in Y(t)$ such that $Y(t)$ is the flow of the vector field $\mathbf{f}$. In addition, assume that the volume of $\Xi(t)$ is $V(t)$. Applying Liouville's theorem as in [37], we obtain

$$
\dot{V}(t)=\int_{\Xi(t)}(\nabla \cdot \mathbf{f}) \prod_{l=1}^{9} d v_{l} .
$$

The vector field $\mathbf{f}$ of (9) has the following divergence:

$$
\begin{aligned}
\nabla \cdot \mathbf{f} & =\sum_{l=1}^{9} \frac{\partial \dot{v}_{l}(t)}{\partial v_{l}(t)} \\
& =-4 \alpha-\beta-4=A,
\end{aligned}
$$

where $A=-4 \alpha-\beta-4$. If $\nabla \cdot \mathbf{f}<0$, then the model is deemed to be dissipative. Consequently, for (4) to be dissipative, we require $4 \alpha+\beta+4>0$. Thus, from (4), we obtain

$$
\begin{aligned}
\dot{V}(t) & =\int_{\Xi(t)}(A) \prod_{l=1}^{9} d v_{l}, \\
& =A V(t) .
\end{aligned}
$$

Integrating (10) gives

$$
V(t)=\exp (A t) V(0)
$$

Because $A<0$, we have that

$$
\lim _{t \rightarrow \infty} V(t)=0,
$$

from which we conclude that the trajectory of this dynamical model (4) forces every volume containing it to diminish to zero. Therefore, we have an attractor that attracts all the trajectories of the new model. From the above, we can demonstrate that the new proposed chaotic model (4) is dissipative.

\subsection{Stability of Equilibria}

The equilibrium points are found by solving $\dot{v}_{1}(t)=0, \dot{v}_{2}(t)=0, \dot{v}_{3}(t)=0, \dot{v}_{4}(t)=0, \dot{v}_{5}(t)=0$, $\dot{v}_{6}(t)=0, \dot{v}_{7}(t)=0, \dot{v}_{8}(t)=0, \dot{v}_{9}(t)=0$, namely 


$$
\begin{aligned}
& 0=\alpha\left(v_{5}-v_{1}\right), \\
& 0=\alpha\left(v_{6}-v_{2}\right), \\
& 0=\alpha\left(v_{7}-v_{3}\right), \\
& 0=\alpha\left(v_{8}-v_{4}\right), \\
& 0=\gamma v_{1}-v_{5}-v_{1} v_{9}, \\
& 0=\gamma v_{2}-v_{6}-v_{2} v_{9}, \\
& 0=\gamma v_{3}-v_{7}-v_{3} v_{9}, \\
& 0=\gamma v_{4}-v_{8}-v_{4} v_{9}, \\
& 0=-\beta v_{9}+v_{1} v_{5}+v_{2} v_{6}+v_{3} v_{7}+v_{4} v_{8} .
\end{aligned}
$$

Analyzing (12) shows that our proposed model has a trivial equilibrium point at $E_{0}=(0,0, \ldots, 0)$ and a nontrivial equilibrium point represented by

$$
v_{1}^{2}+v_{2}^{2}+v_{3}^{2}+v_{4}^{2}=-\beta(\gamma-1) .
$$

\section{Stability analysis of $E_{0}$}

To study the stability of the trivial equilibrium point $E_{0}$, we require its Jacobian matrix

$$
J_{E_{0}}=\left(\begin{array}{ccccccccc}
-\alpha & 0 & 0 & 0 & \alpha & 0 & 0 & 0 & 0 \\
0 & -\alpha & 0 & 0 & 0 & \alpha & 0 & 0 & 0 \\
0 & 0 & -\alpha & 0 & 0 & 0 & \alpha & 0 & 0 \\
0 & b & 0 & -\alpha & 0 & 0 & 0 & \alpha & 0 \\
\gamma & 0 & 0 & 0 & -1 & 0 & 0 & 0 & 0 \\
0 & \gamma & 0 & 0 & 0 & -1 & 0 & 0 & 0 \\
0 & 0 & \gamma & 0 & 0 & 0 & -1 & 0 & 0 \\
0 & 0 & 0 & \gamma & 0 & 0 & 0 & -1 & 0 \\
0 & 0 & 0 & 0 & 0 & 0 & 0 & 0 & -\beta
\end{array}\right)
$$

and its characteristic polynomial

$$
(-\mu-\beta)\left(-\mu^{2}+(-\alpha-1) \mu+\alpha(\gamma-1)\right)^{4}=0,
$$

whose eigenvalues are

$$
\begin{aligned}
\mu_{1} & =-\beta, \\
\mu_{2,3,4,5} & =-\frac{1}{2} \alpha-\frac{1}{2}+\frac{1}{2} \sqrt{\alpha^{2}+4 \alpha \gamma-2 \alpha+1}, \\
\mu_{6,7,8,9} & =-\frac{1}{2} \alpha-\frac{1}{2}-\frac{1}{2} \sqrt{\alpha^{2}+4 \alpha \gamma-2 \alpha+1} .
\end{aligned}
$$

Therefore, the trivial fixed point is stable if

$$
\beta>0, \alpha<0, \gamma<1
$$

and unstable otherwise.

Stability of non-isolated equilibria

The non-isolated equilibria are given by

$$
v_{1}^{2}+v_{2}^{2}+v_{3}^{2}+v_{4}^{2}=-\beta(\gamma-1)
$$


and the nontrivial fixed points can be written as

$$
\begin{aligned}
E_{\theta}= & \left( \pm \sqrt{-\beta(\gamma-1)-\left(v_{2}^{2}(t)+v_{3}^{2}(t)+v_{4}^{2}(t)\right)}, v_{2}(t), v_{3}(t), v_{4}(t),\right. \\
& \left. \pm \sqrt{-\beta(\gamma-1)-\left(v_{2}^{2}(t)+v_{3}^{2}(t)+v_{4}^{2}(t)\right)}, v_{2}(t), v_{3}(t), v_{4}(t), \gamma-1\right) .
\end{aligned}
$$

We set

$$
\begin{aligned}
& v_{1}=v_{5}=\sqrt{-\beta(\gamma+1)} \cos \theta_{1}, \\
& v_{2}=v_{6}=\sqrt{-\beta(\gamma+1)} \sin \theta_{1}, \\
& v_{3}=v_{7}=\sqrt{2 \beta} \cos \theta_{2}, \\
& v_{4}=v_{8}=\sqrt{2 \beta} \sin \theta_{2},
\end{aligned}
$$

where $\theta_{1}, \theta_{2} \in[0,2 \pi]$, and we consider the Jacobian matrix of (4) at the nontrivial fixed point $E_{\theta}$ to study the stability of the latter:

$$
J_{E_{\theta}}=\left[\begin{array}{ccccccccc}
-\alpha & 0 & 0 & 0 & \alpha & 0 & 0 & 0 & 0 \\
0 & -\alpha & 0 & 0 & 0 & \alpha & 0 & 0 & 0 \\
0 & 0 & -\alpha & 0 & 0 & 0 & \alpha & 0 & 0 \\
0 & 0 & 0 & -\alpha & 0 & 0 & 0 & \alpha & 0 \\
1 & 0 & 0 & 0 & -1 & 0 & 0 & 0 & -L_{c_{1}} \\
0 & 1 & 0 & 0 & 0 & -1 & 0 & 0 & -L_{s_{1}} \\
0 & 0 & 1 & 0 & 0 & 0 & -1 & 0 & -L_{c_{2}} \\
0 & 0 & 0 & 1 & 0 & 0 & 0 & -1 & -L_{s_{2}} \\
L_{c_{1}} & L_{s_{1}} & L_{c_{2}} & L_{s_{2}} & L_{c_{1}} & L_{s_{1}} & L_{c_{2}} & L_{s_{2}} & -\beta
\end{array}\right],
$$

where $L_{s_{1}}=\sqrt{-\beta(\gamma+1)} \sin \theta_{1}, L_{c_{1}}=\sqrt{-\beta(\gamma+1)} \cos \theta_{1}, L_{s_{2}}=\sqrt{2 \beta} \sin \theta_{2}$, and $L_{c_{2}}=\sqrt{2 \beta} \cos \theta_{2}$. We compute the characteristic polynomial as

$$
\left[\mu^{3}+(\beta+\alpha+1) \mu^{2}+\beta(\alpha-\gamma+2) \mu+2 \beta \alpha(1-\gamma)\right](\alpha+\mu+1)^{3} \mu^{3}=0 .
$$

By applying the Routh-Hurwitz stability criterion [38], the necessary and sufficient conditions for the stability of the nontrivial fixed point $E_{\theta}$ are $\varepsilon_{1}>0, \varepsilon_{2}>0$, and $\varepsilon_{1} \varepsilon_{2}>\varepsilon_{3}$, where

$$
\varepsilon_{1}=\beta+\alpha+1, \varepsilon_{2}=\beta(\alpha-\gamma+2), \varepsilon_{3}=2 \beta \alpha(1-\gamma) .
$$

Therefore, the nontrivial fixed point is stable under the following conditions:

$$
\alpha>-1, \beta+\alpha>-1, \gamma-\alpha<2, \beta>\frac{\gamma-\alpha^{2}-\alpha \gamma-\alpha-2}{\alpha-\gamma+2} .
$$

\subsection{Lyapunov Exponents}

Model (4) can be recast in a different mathematical form as

$$
\dot{\mathbf{x}}(t)=\Phi(\mathbf{v}(t) ; \eta)
$$

such that $\mathbf{v}(t)=\left[v_{1}(t), v_{2}(t), \ldots, v_{9}(t)\right]^{T}$ represents the state-space vector $\Phi(t)=$ $\left[\Phi_{1}(t), \Phi_{2}(t), \ldots, \Phi_{9}(t)\right]^{T}$, where $[\ldots]^{T}$ symbolizes matrix transpose and the set of parameters is arranged in $\eta$. A small deviation $\delta \mathbf{x}_{(t)}$ from the trajectory $\mathbf{x}_{(t)}$ is calculated by

$$
\delta \dot{\mathbf{x}}(t)=J_{l k}(\mathbf{v}(t) ; \eta) \delta \mathbf{v} \text { where } 1 \leq l, k \leq 9
$$


and the Jacobian matrix $J_{l k}\left(\mathbf{v}_{(t)} ; \eta\right)=\frac{\partial \Phi_{l}}{\partial \mathbf{v}_{k}}$ is

$$
J_{l k}=\left(\begin{array}{ccccccccc}
-\alpha & 0 & 0 & 0 & \alpha & 0 & 0 & 0 & 0 \\
0 & -\alpha & 0 & 0 & 0 & \alpha & 0 & 0 & 0 \\
0 & 0 & -\alpha & 0 & 0 & 0 & \alpha & 0 & 0 \\
0 & 0 & 0 & -\alpha & 0 & 0 & 0 & \alpha & 0 \\
\gamma-v_{9} & 0 & 0 & 0 & -1 & 0 & 0 & 0 & -v_{1} \\
0 & \gamma-v_{9} & 0 & 0 & 0 & -1 & 0 & 0 & -v_{2} \\
0 & 0 & \gamma-v_{9} & 0 & 0 & 0 & -1 & 0 & -v_{3} \\
0 & 0 & 0 & \gamma-v_{9} & 0 & 0 & 0 & -1 & -v_{4} \\
v_{5} & v_{6} & v_{7} & v_{8} & v_{1} & v_{2} & v_{3} & v_{4} & -\beta
\end{array}\right)
$$

The Lyapunov exponents [39] $\lambda_{k}$ of our system are calculated as

$$
\lambda_{l}=\lim _{t \rightarrow \infty} \frac{1}{t} \log \frac{\|\delta \mathbf{v}(t)\|}{\|\delta \mathbf{v}(0)\|} ; l=1,2, \ldots, 9 .
$$

To evaluate $\lambda_{k}$, we use the fourth-order Runge-Kutta method to calculate (16) and (17) numerically. For $\alpha=25, \gamma=48$, and $\beta=5$ and with initial conditions $v_{p}(0)=p(1 \leq p \leq 9)$, the Lyapunov exponents are computed as $\lambda_{1}=2.45, \lambda_{2}=0, \lambda_{3}=0, \lambda_{4}=0, \lambda_{5}=0, \lambda_{6}=-37.5, \lambda_{7}=-37.5$, $\lambda_{8}=-37.5$, and $\lambda_{9}=-47.1$. Under this choice of $\alpha, \gamma$, and $\beta$, these results show that (4) has one positive Lyapunov exponent and thus chaotic behavior, as supported by the many previous papers that have shown that chaotic models have one positive Lyapunov exponent. As indicated by the Kaplan-Yorke conjecture [40], the dimension of the Lyapunov space of the attractor of (4) is characterized as

$$
D=\Omega+\frac{\sum_{k=1}^{\Omega} \lambda_{k}}{\left|\lambda_{\Omega+1}\right|}
$$

where $\Omega$ is the largest integer that satisfies $\sum_{k=1}^{\Omega} \lambda_{k}>0$ and $\sum_{k=1}^{\Omega+1} \lambda_{k}<0$. Applying (19), the Lyapunov dimension of our chaotic attractor is calculated as $D=5+\frac{\lambda_{1}+\lambda_{2}+\lambda_{3}+\lambda_{4}+\lambda_{5}}{\left|\lambda_{6}\right|} \approx 5.065333$.

Fix $\beta, \gamma$ and $\alpha$ is shift where $\alpha \in[0,35]$

The Lyapunov exponents $\lambda_{1}, \lambda_{2}, \ldots, \lambda_{9}$ are determined using (18). From the behavior of the Lyapunov exponents as shown in Figure 3, (4) fluctuates between fixed-point and chaotic attractors. From Figure 3, we have the following two cases. For $\alpha \in[0,8],(4)$ has a fixed-point attractor for which all the Lyapunov exponents are negative. For $\alpha \in(8,35],(4)$ has a chaotic attractor for which one Lyapunov exponent is positive. The same analysis as described here for parameter $\alpha$ was conducted for $\beta$ and $\gamma$, and chaotic solutions with one positive Lyapunov exponent were again obtained. From these results, the solutions of (4) can be classified as follows in Table 1.

Table 1. Solutions of system (4).

\begin{tabular}{cccccccccc}
\hline$\lambda_{1}$ & $\lambda_{2}$ & $\lambda_{3}$ & $\lambda_{4}$ & $\lambda_{5}$ & $\lambda_{6}$ & $\lambda_{7}$ & $\lambda_{8}$ & $\lambda_{9}$ & Attractor \\
\hline- & - & - & - & - & - & - & - & - & Fixed point \\
+ & 0 & 0 & 0 & 0 & - & - & - & - & Chaotic \\
\hline
\end{tabular}



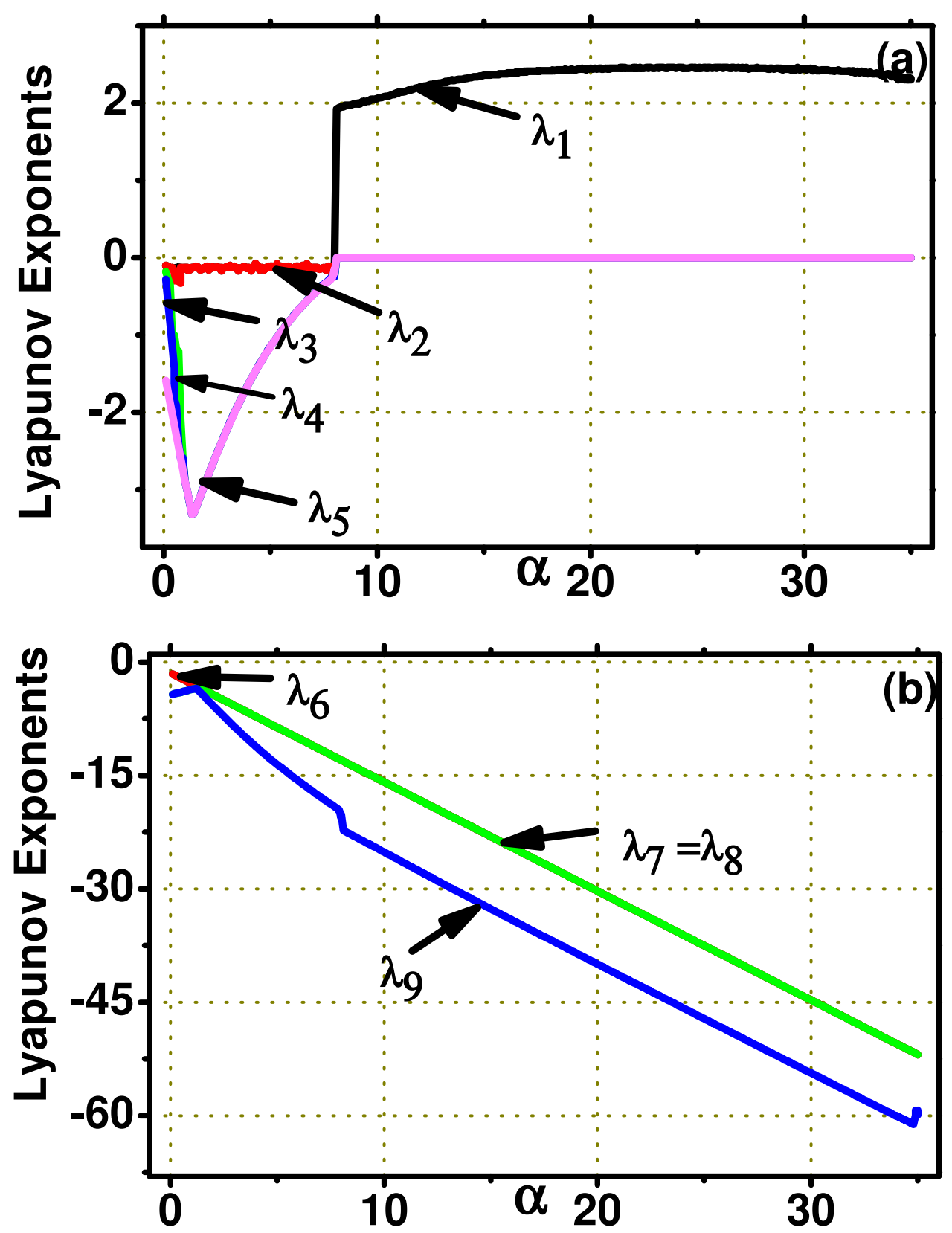

Figure 3. For $\gamma=48, \beta=5$, and vary $\alpha$ with $v_{p}(0)=p, 1 \leq p \leq 9$ as an initial conditions. (a) Lyapunov exponents of model (4): $\lambda_{1}, \lambda_{2}, \lambda_{3}, \lambda_{4}, \lambda_{5}$; (b) Lyapunov exponents of model (4): $\lambda_{6}, \lambda_{7}, \lambda_{8}, \lambda_{9}$.

\subsection{Bifurcation Diagram of (4)}

A bifurcation diagram shows how a deterministic system responds when one of its parameters is varied [41]; the horizontal axis represents the value of the parameter being varied and the vertical axis records the attractor's activity. As such, a bifurcation diagram is a means of picturing how changing the selected parameter changes the model's behavior. In this place, to ensure the dynamic behavior of model (4), the bifurcation diagram is used. To establish further that (4) behaves chaotically, bifurcation diagrams obtained by varying $\alpha$ are plotted in Figure 4 for $\alpha \in[0,35]$. Figure 4a shows the $\left(\alpha, v_{3}(t)\right)$ bifurcation diagram, which shows that (4) has a chaotic attractor when $\alpha \in(8,35]$. Figure $4 \mathrm{~b}$ shows the $\left(\alpha, v_{8}(t)\right)$ bifurcation diagram. We used the Mathematica 7 software to solve (4) numerically in a few cases, and the results are completely similar with the past results in Section 3.6. For example, 
for $\beta=5$ and $\gamma=48$ with $v_{p}(0)=p(1 \leq p \leq 9)$ as the initial conditions, (4) has a fixed point when $\alpha=4$ and 7 , for which all the Lyapunov exponents are negative (see Figure $5 \mathrm{a}, \mathrm{b}$ ). In Figure $5 \mathrm{c}, \mathrm{d}$ for $\alpha=25$ and 30, the arrangement is chaotic with one positive Lyapunov exponent.
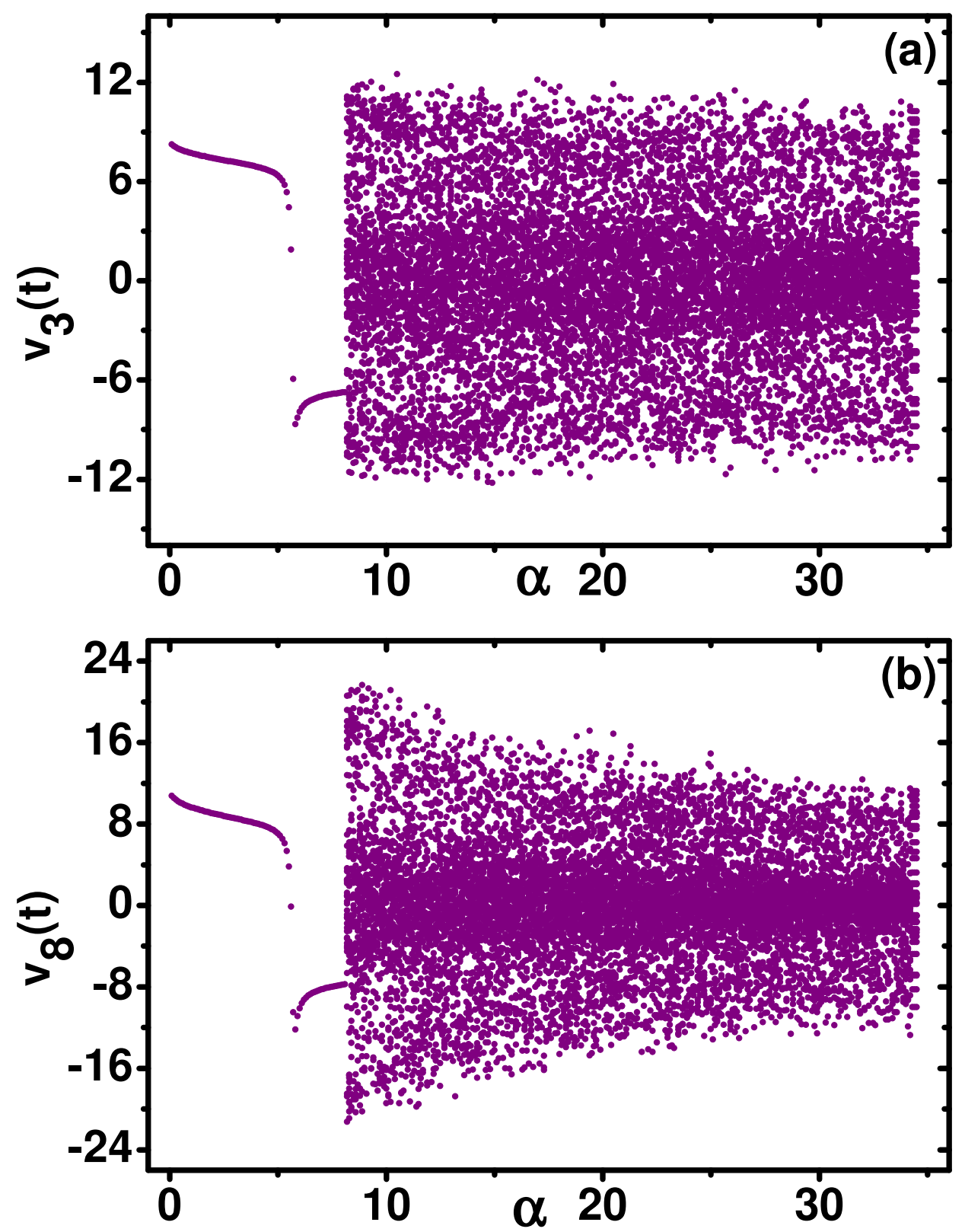

Figure 4. For $\gamma=48, \beta=5$ and vary $\alpha$ with the sama initial conditions in Figure 3. (a) bifurcation diagrams in $\left(\alpha, v_{3}(t)\right)$ plane; (b) bifurcation diagrams in $\left(\alpha, v_{8}(t)\right)$ plane. 
(a)

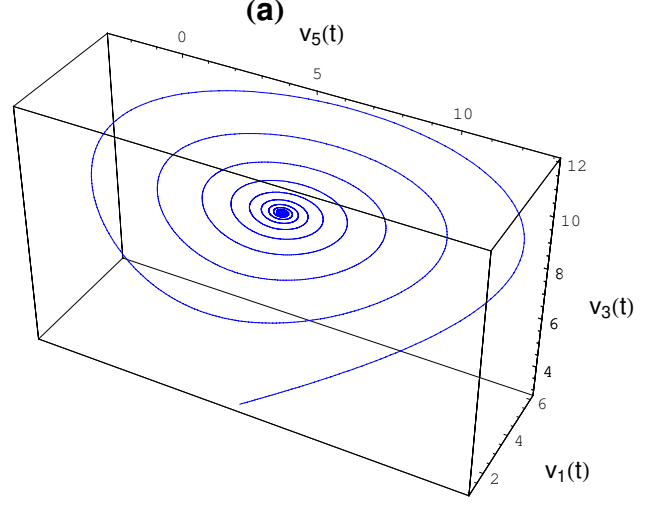

(c) $\quad v_{5}(t)$

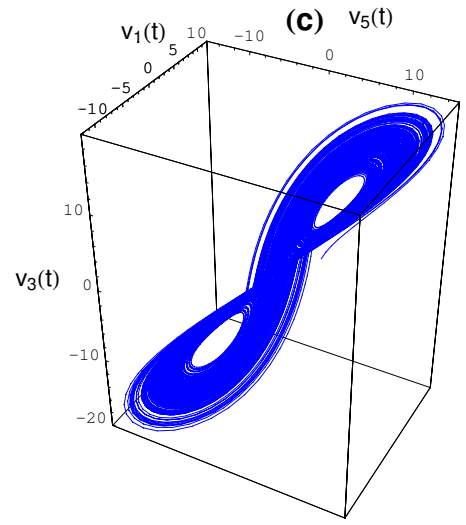

(b)

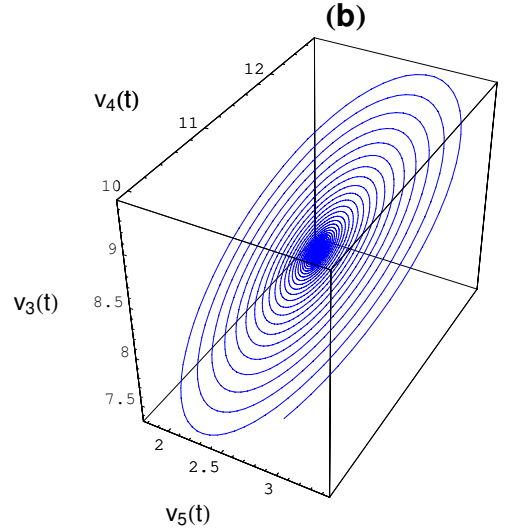

(d) $v_{5}(t)$

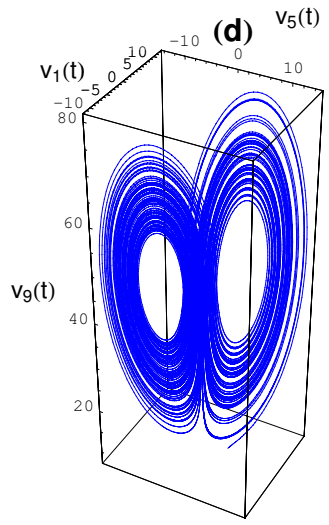

Figure 5. Solutions of model (4) for $\gamma=48, \beta=5$, and vary $\alpha$ with the same initial conditions in Figure 3. (a) fixed point, $\alpha=4$, in $\left(v_{5}(t), v_{3}(t), v_{1}(t)\right)$; (b) fixed point, $\alpha=7$, in $\left(v_{5}(t), v_{3}(t), v_{4}(t)\right)$; (c) chaotic attractors, $\alpha=25$, in $\left(v_{5}(t), v_{1}(t), v_{3}(t)\right)$; (d) chaotic attractors, $\alpha=30$, in $\left(v_{5}(t), v_{1}(t)\right.$, $\left.v_{9}(t)\right)$.

\section{Simulink Simulation and Design of Electronic Circuit}

Here, we construct an electronic circuit for the studied system and compare its simulation results with those obtained numerically in Section 3. Figure 6 shows the designed electronic version of (4); the values of all the resistors and capacitors realize the system parameters given in Section 3, namely $\alpha=25, \gamma=48$, and $\beta=5$. This circuit contains nine different channels that realize the state variables $v_{1}(t), v_{2}(t), \ldots, v_{9}(t)$. In addition, each channel consists of a summer, a multiplier, an integrator, and an inverter, all of which are designed using an operational amplifier of type TL082 and an operational multiplier of type AD633 with a $\pm 15 \mathrm{~V}$ voltage supply. Figure 7 depicts the Simulink simulation with the real variables $v_{1}(t), v_{2}(t), \ldots, v_{9}(t)$. The simulation results shown in Figure 8 are similar to those obtained from the numerical solutions shown in Figure 5. 


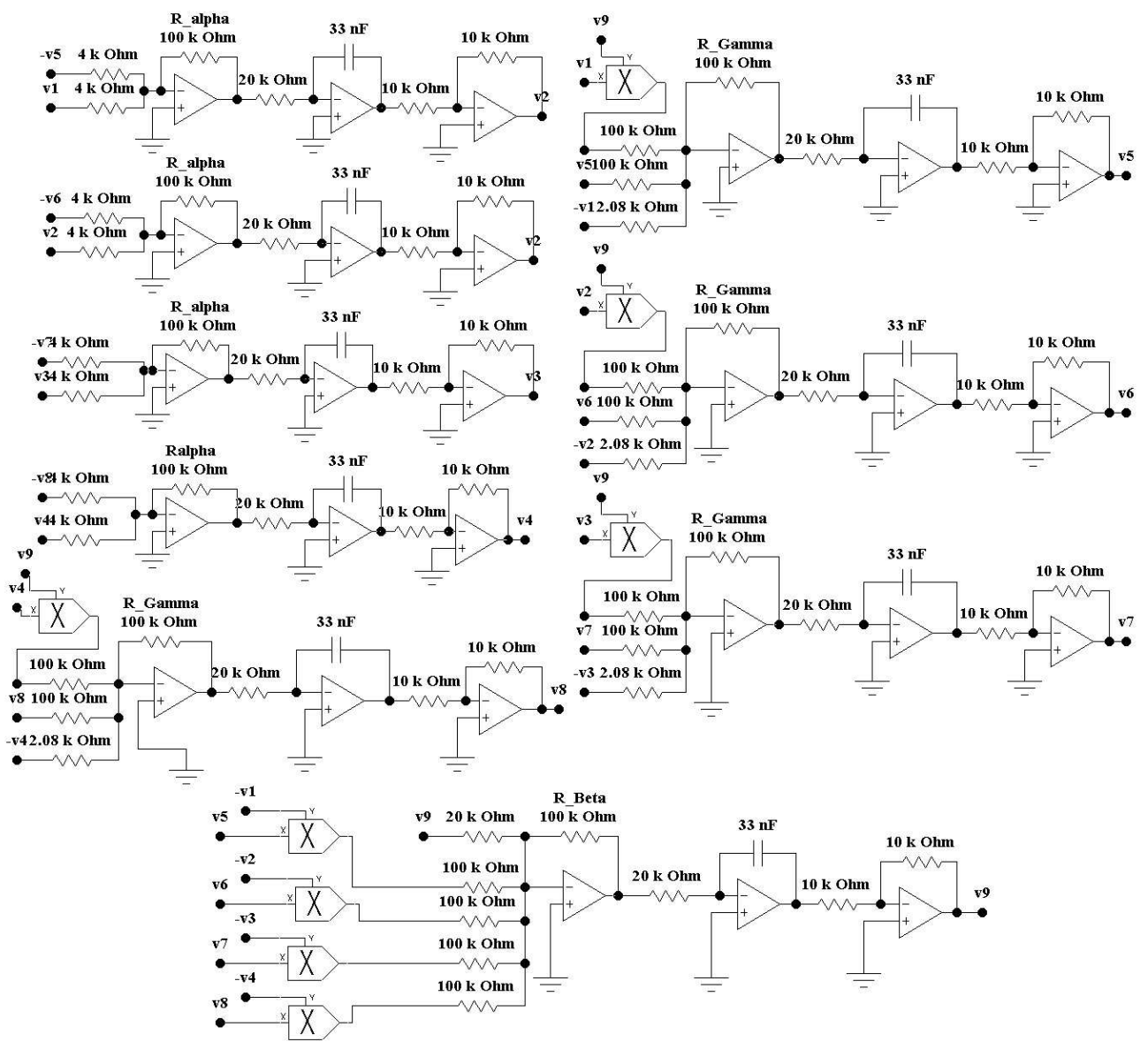

Figure 6. The electronic circuit diagram of the studied system (4).

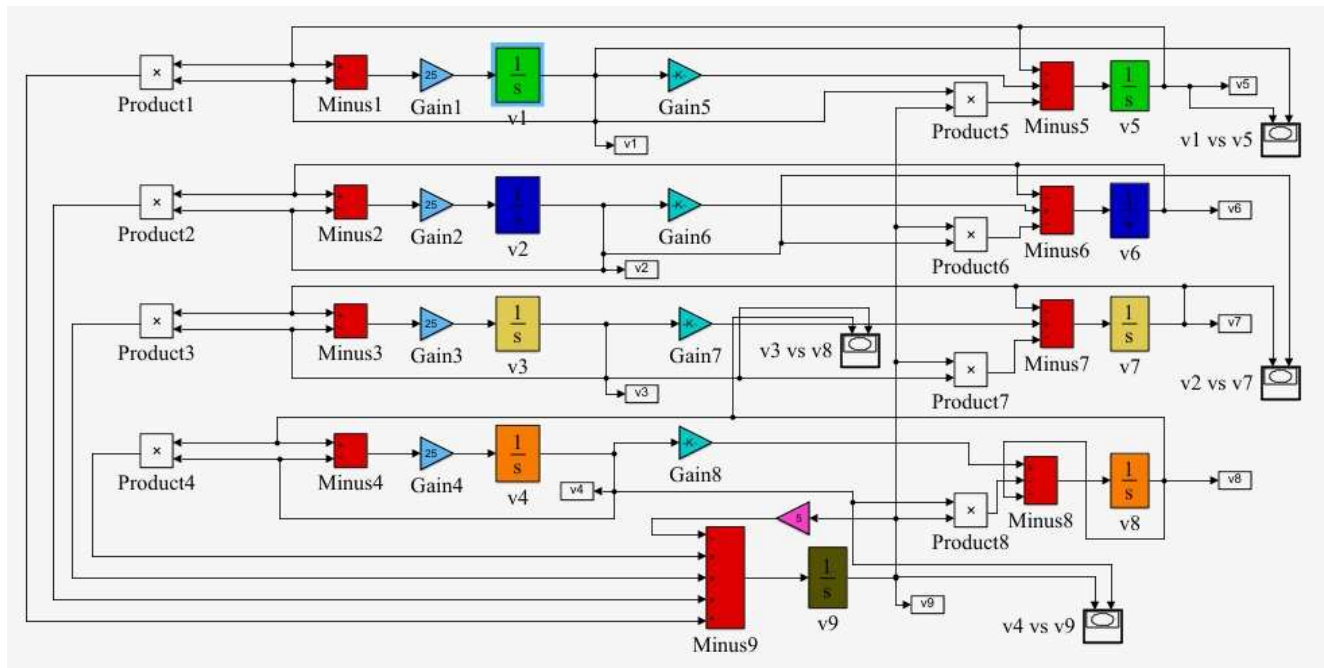

Figure 7. The simulation graph of the studied system (4) using Matlab/Simulink. 

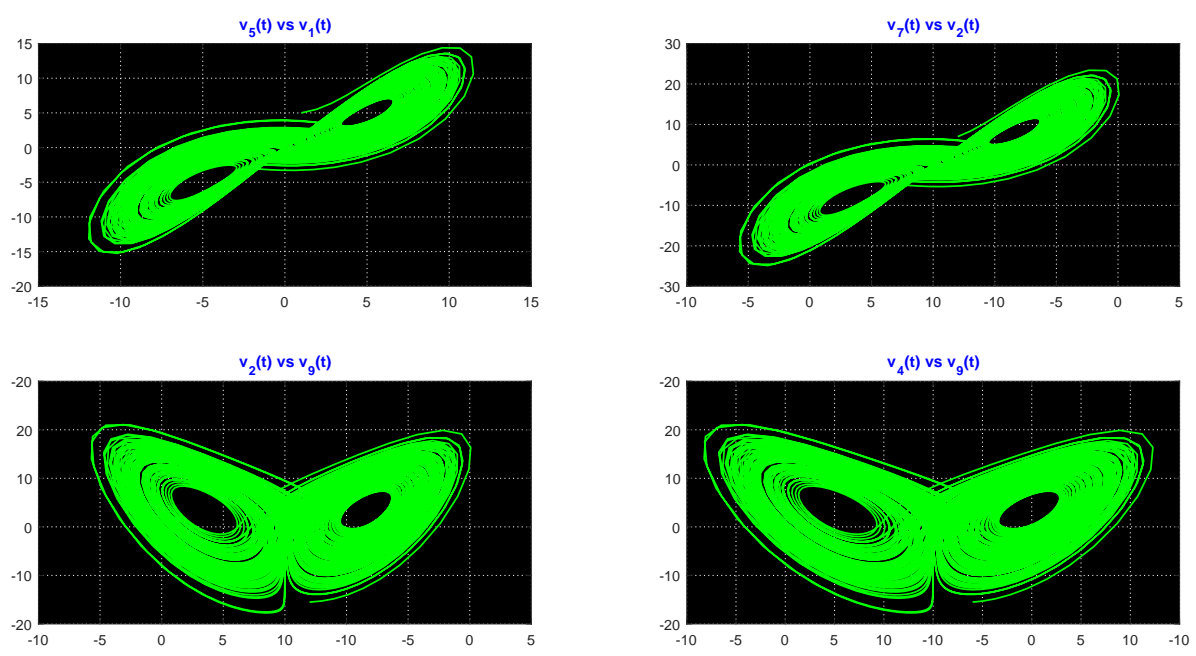

Figure 8. Simulation response of the chaotic attractor of system (4): (left-top) $\left(v_{5}(t), v_{1}(t)\right)$ phase plane; (right-top) $\left(v_{7}(t), v_{2}(t)\right)$ phase plane; (left-bottom) $\left(v_{2}(t)-v_{9}(t)\right)$ phase plane; (right-bottom) $\left(v_{4}(t)-v_{9}(t)\right)$ phase plane.

\section{AAS for Chaotic Quaternion Nonlinear Systems}

\subsection{Chaotic Quaternion Nonlinear Systems}

Several engineering applications use the quaternion system, such as communications. In communications, quaternions are used to quadruple the number of variables, thereby allowing more content to be communicated safely. If a quaternion dynamical system is sensitive to its initial conditions with long-term cyclic deterministic conduct, then it is called a chaotic system. A chaotic quaternion attractor has at least one positive Lyapunov exponent. To ensure that the system has dissipative behavior, its Lyapunov exponents must sum to a negative number. Compared to chaotic non-quaternion systems, chaotic quaternion systems are considered to be more complicated with more-unstable manifolds. Because chaotic quaternion systems have high security, high efficiency, and high capacity, they have several wide-ranging applications, such as in biological systems, neural networks, nonlinear circuits, lasers, and secure communications.

Here, the nonlinear chaotic quaternion system has the form

$$
\dot{\mathbf{x}}=\mathbf{A x}+\mathbf{f}(\mathbf{x}),
$$

where $\mathbf{x}$ is the vector of quaternion state variables $x_{1}, x_{2}, \ldots, x_{n}$. Furthermore, we have that $\mathbf{x}=\mathbf{x}^{r}+$ $i \mathbf{x}^{i}+j \mathbf{x}^{j}+k \mathbf{x}^{k}, \mathbf{x}^{r}=\left[v_{1}, v_{5}, \ldots, v_{4 n-3}\right]^{T}, \mathbf{x}^{i}=\left[v_{2}, v_{6}, \ldots, v_{4 n-2}\right]^{T}, \mathbf{x}^{j}=\left[v_{3}, v_{7}, \ldots, v_{4 n-1}\right]^{T}$, and $\mathbf{x}^{k}=$ $\left[v_{4}, v_{8}, \ldots, v_{4 n}\right]^{T}$, with $i^{2}=j^{2}=k^{2}=1$. Moreover, $T$ symbolizes matrix transpose, $\mathbf{A} \in R^{n \times n}$ is the quaternion (or real) matrix that contains the system parameters, $\mathbf{f}=\left[f_{1}, f_{2}, \ldots, f_{n}\right]^{T}$ is a vector of nonlinear quaternion functions, and superscripts $r$ and $i, j, k$ denote the real and imaginary parts, respectively, of the quaternion state vector $\mathbf{x}$. In this work, the AAS of two similar systems of form (20) with predefined parameters is investigated by designing a control procedure.

\subsection{Design Plan for AAS Quaternion Controller}

Two similar chaotic quaternion nonlinear systems are considered. The first one is the drive or master system (denoted by the subscript $d$ ) defined as

$$
\dot{\mathbf{x}}_{d}=\dot{\mathbf{x}}_{d}^{r}+i \dot{\mathbf{x}}_{d}^{i}+j \dot{\mathbf{x}}_{d}^{j}+k \dot{\mathbf{x}}_{d}^{k}=\mathbf{A} \mathbf{x}_{d}+\mathbf{f}\left(\mathbf{x}_{d}\right) .
$$


The second one is the controlled response system (denoted by the subscript $r$ ) defined as

$$
\dot{x}_{r}=\dot{\mathbf{x}}_{r}^{r}+i \dot{\mathbf{x}}_{r}^{i}+j \dot{\mathbf{x}}_{r}^{j}+k \dot{\mathbf{x}}_{r}^{k}=\mathbf{A} \mathbf{x}_{r}+\mathbf{f}\left(\mathbf{x}_{r}\right)+\mathbf{L},
$$

where the additive quaternion controller $\mathbf{L}=\mathbf{L}^{r}+i \mathbf{L}^{i}+j \mathbf{L}^{j}+k \mathbf{L}^{k}=\left[L_{1}, L_{2}, \ldots, L_{n}\right]^{T}$, with $\mathbf{L}^{r}=$ $\left[u_{1}, u_{5}, \ldots, u_{4 n-3}\right]^{T}, \mathbf{L}^{i}=\left[u_{2}, u_{6}, \ldots, u_{4 n-2}\right]^{T}, \mathbf{L}^{j}=\left[u_{3}, u_{7}, \ldots . ., u_{4 n-1}\right]^{T}$, and $\mathbf{L}^{k}=\left[u_{4}, u_{8}, \ldots ., u_{4 n}\right]^{T}$.

Definition 1. Two identical quaternion dynamical systems coupled in a drive-response configuration can offer $A A S$ if there exists a vector of the quaternion error function defined as

$$
\mathbf{e}=\mathbf{e}^{r}+i \mathbf{e}^{i}+j \mathbf{e}^{j}+k \mathbf{e}^{k}=\lim _{t \rightarrow \infty}\left\|\mathbf{x}_{r}(t)+\mathbf{x}_{d}(t+\tau)\right\|=0,
$$

where $\mathbf{e}=\left[e_{1}, e_{2}, \ldots, e_{n}\right]^{T}, \mathbf{x}_{d}(t)$ and $\mathbf{x}_{r}(t)$ are the state quaternion vectors of the drive and response systems whether they are identical, respectively, $\mathbf{e}^{r}=\lim _{t \rightarrow \infty}\left\|\mathbf{x}_{r}^{r}(t)+\mathbf{x}_{d}^{r}(t+\tau)\right\|=0, \mathbf{e}^{i}=\lim _{t \rightarrow \infty}\left\|\mathbf{x}_{r}^{i}(t)+\mathbf{x}_{d}^{i}(t+\tau)\right\|=$ $0, \mathbf{e}^{j}=\lim _{t \rightarrow \infty}\left\|\mathbf{x}_{r}^{j}(t)+\mathbf{x}_{d}^{j}(t+\tau)\right\|=0, \mathbf{e}^{k}=\lim _{t \rightarrow \infty}\left\|\mathbf{x}_{r}^{k}(t)+\mathbf{x}_{d}^{k}(t+\tau)\right\|=0$, and $\mathbf{e}^{r}=\left[e_{v_{1}}, e_{v_{5}}, \ldots . . e_{v_{(4 n-3)}}\right]$, $\mathbf{e}^{i}=\left[e_{v_{2}}, e_{v_{6}}, \ldots . e_{v_{(4 n-2)}}\right], \mathbf{e}^{j}=\left[e_{v_{3}}, e_{v_{7}}, \ldots . e_{v_{(4 n-1)}}\right], \mathbf{e}^{k}=\left[e_{v_{4}}, e_{v_{8}}, \ldots . e_{v_{(4 n)}}\right]$, and $\tau$ is the positive time anticipating.

Remark 1. When $\tau=0$ in (23), we define there the anti-synchronization (AS) between systems (21) and (22) [42].

Remark 2. If we define $\mathbf{e}=\mathbf{e}^{r}+i \mathbf{e}^{i}+j \mathbf{e}^{j}+k \mathbf{e}^{k}=\lim _{t \rightarrow \infty}\left\|\mathbf{x}_{r}(t)-\mathbf{x}_{d}(t+\tau)\right\|=0$, and $\tau=0$, then we obtain the complete synchronization (CS) of systems (21) and (22) [43].

Remark 3. We obtain the lag synchronization (LS) of systems (21) and (22), when we set $\mathbf{e}=\mathbf{e}^{r}+i \mathbf{e}^{i}+j \mathbf{e}^{j}+$ $k \mathbf{e}^{k}=\lim _{t \rightarrow \infty}\left\|\mathbf{x}_{r}(t)-\mathbf{x}_{d}(t-\tau)\right\|=0$, and $\tau>0$, then [44].

Remark 4. If we define $\mathbf{e}=\mathbf{e}^{r}+i \mathbf{e}^{i}+j \mathbf{e}^{j}+k \mathbf{e}^{k}=\lim _{t \rightarrow \infty}\left\|\mathbf{x}_{r}(t)-\mathbf{x}_{d}(t+\tau)\right\|=0$, then we obtain the anticipate synchronization (AnS) of systems (21) and (22) [45].

Theorem 1. If a nonlinear controller is designed as:

$$
\begin{aligned}
\mathbf{L}= & \mathbf{L}^{r}+i \mathbf{L}^{i}+j \mathbf{L}^{j}+k \mathbf{L}^{k}=-\mathbf{A} \mathbf{x}_{r}(t)-\mathbf{f}\left(\mathbf{x}_{r}(t)\right)-\mathbf{A} \mathbf{x}_{d}(t+\tau)-\mathbf{f}\left(\mathbf{x}_{d}(t+\tau)\right)-\zeta \mathbf{e} \\
= & -\mathbf{A} \mathbf{x}_{r}^{r}(t)-\mathbf{f}^{r}\left(\mathbf{x}_{r}(t)\right)-\mathbf{A} \mathbf{x}_{d}^{r}(t+\tau)-\mathbf{f}^{r}\left(\mathbf{x}_{d}(t+\tau)\right)-\zeta \mathbf{e}^{r} \\
& +i\left[-\mathbf{A} \mathbf{x}_{r}^{i}(t)-\mathbf{f}^{i}\left(\mathbf{x}_{r}(t)\right)-\mathbf{A} \mathbf{x}_{d}^{i}(t+\tau)-\mathbf{f}^{i}\left(\mathbf{x}_{d}(t+\tau)\right)-\zeta \mathbf{e}^{i}\right] \\
& +j\left[-\mathbf{A} \mathbf{x}_{r}^{j}(t)-\mathbf{f}^{j}\left(\mathbf{x}_{r}(t)\right)-\mathbf{A} \mathbf{x}_{d}^{j}(t+\tau)-\mathbf{f}^{j}\left(\mathbf{x}_{d}(t+\tau)\right)-\zeta \mathbf{e}^{j}\right] \\
& +k\left[-\mathbf{A} \mathbf{x}_{r}^{k}(t)-\mathbf{f}^{k}\left(\mathbf{x}_{r}(t)\right)-\mathbf{A} \mathbf{x}_{d}^{k}(t+\tau)-\mathbf{f}^{k}\left(\mathbf{x}_{d}(t+\tau)\right)-\zeta \mathbf{e}^{k}\right],
\end{aligned}
$$

then the AAS is achieved among the response system (22) and the drive system (21) asymptotically, where $\zeta>0$.

Proof. From the definition of AAS,

$$
\mathbf{e}=\mathbf{e}^{r}+i \mathbf{e}^{i}+j \mathbf{e}^{j}+k \mathbf{e}^{k}=\mathbf{x}_{r}(t)+\mathbf{x}_{d}(t+\tau) .
$$

Thus,

$$
\begin{aligned}
\dot{\mathbf{e}}= & \dot{\mathbf{e}}^{r}+i \dot{\mathbf{e}}^{i}+j \dot{\mathbf{e}}^{j}+k \dot{\mathbf{e}}^{k}=\mathbf{x}_{r}(t)+\mathbf{x}_{d}(t+\tau)=\mathbf{x}_{r}^{r}(t)+\mathbf{x}_{d}^{r}(t+\tau) \\
& +i\left[\mathbf{x}_{r}^{i}(t)+\mathbf{x}_{d}^{i}(t+\tau)\right]+j\left[\mathbf{x}_{r}^{j}(t)+\mathbf{x}_{d}^{j}(t+\tau)\right]+k\left[\mathbf{x}_{r}^{k}(t)+\mathbf{x}_{d}^{k}(t+\tau)\right] .
\end{aligned}
$$


We always perceive the dynamic error quaternion system from chaotic quaternion systems (21) and (22) as obeys

$$
\begin{aligned}
\dot{\mathbf{e}}= & \dot{\mathbf{e}}^{r}+i \dot{\mathbf{e}}^{i}+j \dot{\mathbf{e}}^{j}+k \dot{\mathbf{e}}^{k}=\mathbf{A} \mathbf{x}_{r}^{r}(t)+\mathbf{f}^{r}\left(\mathbf{x}_{r}(t)\right)+\mathbf{A} \mathbf{x}_{d}^{r}(t+\tau)+\mathbf{f}^{r}\left(\mathbf{x}_{d}(t+\tau)\right)+\mathbf{L}^{r} \\
& i\left[\mathbf{A} \mathbf{x}_{r}^{i}(t)+\mathbf{f}^{i}\left(\mathbf{x}_{r}(t)\right)+\mathbf{A} \mathbf{x}_{d}^{i}(t+\tau)+\mathbf{f}^{i}\left(\mathbf{x}_{d}(t+\tau)\right)+\mathbf{L}^{i}\right]+j\left[\mathbf{A} \mathbf{x}_{r}^{j}(t)+\mathbf{f}^{j}\left(x_{r}(t)\right)\right. \\
& \left.+\mathbf{A} \mathbf{x}_{d}^{j}(t+\tau)+\mathbf{f}^{j}\left(\mathbf{x}_{d}(t+\tau)\right)+\mathbf{L}^{j}\right]+k\left[\mathbf{A} \mathbf{x}_{r}^{k}(t)+\mathbf{f}^{k}\left(\mathbf{x}_{r}(t)\right)+\mathbf{A} \mathbf{x}_{d}^{k}(t+\tau)\right. \\
& \left.+\mathbf{f}^{k}\left(\mathbf{x}_{d}(t+\tau)\right)+\mathbf{L}^{k}\right] .
\end{aligned}
$$

The error quaternion system is actually drafted by separating the real and imaginary parts in (27):

$$
\begin{aligned}
\dot{\mathbf{e}}^{r} & =\mathbf{A} \mathbf{x}_{r}^{r}(t)+\mathbf{f}^{r}\left(x_{r}(t)\right)+\mathbf{A} \mathbf{x}_{d}^{r}(t+\tau)+\mathbf{f}^{r}\left(\mathbf{x}_{d}(t+\tau)\right)+\mathbf{L}^{r}, \\
\dot{\mathbf{e}}^{i} & =\mathbf{A} \mathbf{x}_{r}^{i}(t)+\mathbf{f}^{i}\left(x_{r}(t)\right)+\mathbf{A} \mathbf{x}_{d}^{i}(t+\tau)+\mathbf{f}^{i}\left(\mathbf{x}_{d}(t+\tau)\right)+\mathbf{L}^{i}, \\
\dot{\mathbf{e}}^{j} & =\mathbf{A} \mathbf{x}_{r}^{j}(t)+\mathbf{f}^{j}\left(x_{r}(t)\right)+\mathbf{A} \mathbf{x}_{d}^{j}(t+\tau)+\mathbf{f}^{j}\left(\mathbf{x}_{d}(t+\tau)\right)+\mathbf{L}^{j}, \\
\dot{\mathbf{e}}^{k} & =\mathbf{A} \mathbf{x}_{r}^{k}(t)+\mathbf{f}^{k}\left(x_{r}(t)\right)+\mathbf{A} \mathbf{x}_{d}^{k}(t+\tau)+\mathbf{f}^{k}\left(\mathbf{x}_{d}(t+\tau)\right)+\mathbf{L}^{k} .
\end{aligned}
$$

When the parameters are positive, the following positive-definite quantity can be applied to define a Lyapunov function for our system as

$$
\begin{aligned}
V(t) & =\frac{1}{2}\left[\left(\mathbf{e}^{r}\right)^{T} \mathbf{e}^{r}+\left(\mathbf{e}^{i}\right)^{T} \mathbf{e}^{i}+\left(\mathbf{e}^{j}\right)^{T} \mathbf{e}^{j}+\left(\mathbf{e}^{k}\right)^{T} \mathbf{e}^{k}\right] \\
& =\frac{1}{2}\left[\sum_{l=1}^{n} e_{v_{4 l-3}}^{2}+\sum_{l=1}^{n} e_{v_{4 l-2}}^{2}+\sum_{l=1}^{n} e_{v_{4 l-1}}^{2}+\sum_{l=1}^{n} e_{v_{4 l}}^{2}\right] .
\end{aligned}
$$

Note that Lyapunov's total time derivative function along the error system trajectory (28) is

$$
\begin{aligned}
\dot{V}(t)= & \left(\dot{\mathbf{e}}^{r}\right)^{T} \mathbf{e}^{r}+\left(\dot{\mathbf{e}}^{i}\right)^{T} \mathbf{e}^{i}+\left(\dot{\mathbf{e}}^{j}\right)^{T} \mathbf{e}^{j}+\left(\dot{\mathbf{e}}^{k}\right)^{T} \mathbf{e}^{k}=\left(\mathbf{A} \mathbf{x}_{r}^{r}(t)+\mathbf{f}^{r}\left(\mathbf{x}_{r}(t)\right)\right. \\
& \left.+\mathbf{A} \mathbf{x}_{d}^{r}(t+\tau)+\mathbf{f}^{r}\left(\mathbf{x}_{d}(t+\tau)\right)+\mathbf{L}^{r}\right)^{T} \mathbf{e}^{r}+\left(\mathbf{A} \mathbf{x}_{r}^{i}(t)+\mathbf{f}^{i}\left(\mathbf{x}_{r}(t)\right)\right. \\
& \left.+\mathbf{A} \mathbf{x}_{d}^{i}(t+\tau)+\mathbf{f}^{i}\left(\mathbf{x}_{d}(t+\tau)\right)+\mathbf{L}^{i}\right)^{T} \mathbf{e}^{i}+\left(\mathbf{A} \mathbf{x}_{r}^{j}(t)+\mathbf{f}^{j}\left(\mathbf{x}_{r}(t)\right)\right. \\
& \left.+\mathbf{A} \mathbf{x}_{d}^{j}(t+\tau)+\mathbf{f}^{j}\left(x_{d}(t+\tau)\right)+\mathbf{L}^{j}\right)^{T} \mathbf{e}^{j}+\left(\mathbf{A} \mathbf{x}_{r}^{k}(t)+\mathbf{f}^{k}\left(x_{r}(t)\right)\right. \\
& \left.+\mathbf{A} \mathbf{x}_{d}^{k}(t+\tau)+\mathbf{f}^{k}\left(\mathbf{x}_{d}(t+\tau)\right)+\mathbf{L}^{k}\right)^{T} \mathbf{e}^{k} .
\end{aligned}
$$

By substituting from (24) regarding $\mathbf{L}^{r}, \mathbf{L}^{i}, \mathbf{L}^{j}$, and $\mathbf{L}^{k}$ in (30), we obtain

$$
\begin{aligned}
\dot{V}(t) & =-\zeta\left[\left(\mathbf{e}^{r}\right)^{T} \mathbf{e}^{r}+\left(\mathbf{e}^{i}\right)^{T} \mathbf{e}^{i}+\left(\mathbf{e}^{j}\right)^{T} \mathbf{e}^{j}+\left(\mathbf{e}^{k}\right)^{T} \mathbf{e}^{k}\right] \\
& =-\zeta\left[\sum_{l=1}^{n} e_{v_{4 l-3}}^{2}+\sum_{l=1}^{n} e_{v_{4 l-2}}^{2}+\sum_{l=1}^{n} e_{v_{4 l-1}}^{2}+\sum_{l=1}^{n} e_{v_{4 l}}^{2}\right] .
\end{aligned}
$$

As per the well-known Lyapunov hypothesis, the system of quaternion errors (27) is asymptotically stable because $V(t)$ is definitely a positive function and its derivative is definitely negative. Thus, $e_{v_{4 n-3}}, e_{v_{4 n-2}}, e_{v_{4 n-1}}$, and $e_{v_{4 n}}$ vanish as $t \rightarrow \infty, l=1,2, \ldots, n$. Therefore, with predicting time, the state of the response side and the drive side will be asymptotically AAS globally. The proof is complete. 


\subsection{Example}

Here, we present the probability and effectiveness of the proposed synchronization as discussed in Section 5.2. The AAS of two similar chaotic quaternion models is studied. In the following, we define the drive and the response systems independently as

$$
\begin{aligned}
& \dot{x}_{d}=\alpha\left(y_{d}-x_{d}\right) \\
& \dot{y}_{d}=\gamma x_{d}-y_{d}-x_{d} z_{d} \\
& \dot{z}_{d}=-\beta z_{d}+\frac{1}{2}\left(y_{d} \bar{x}_{d}+x_{d} \bar{y}_{d}\right)
\end{aligned}
$$

and

$$
\begin{aligned}
& \dot{x}_{r}=\alpha\left(y_{r}-x_{r}\right)+L_{1}, \\
& \dot{y}_{r}=\gamma x_{r}-y_{r}-x_{r} z_{r}+L_{2}, \\
& \dot{z}_{r}=-\beta z_{r}+\frac{1}{2}\left(y_{r} \bar{x}_{r}+x_{r} \bar{y}_{r}\right)+L_{3},
\end{aligned}
$$

where $x_{d}=v_{1 d}+i v_{2 d}+j v_{3 d}+k v_{4 d}, y_{d}=v_{5 d}+i v_{6 d}+j v_{7 d}+k v_{8 d}, z_{d}=v_{9 d}, x_{r}=v_{1 r}+i v_{2 r}+j v_{3 r}+k v_{4 r}$, $y_{r}=v_{5 r}+i v_{6 r}+j v_{7 r}+k v_{8 r}, z_{r}=v_{9 r}$, an overbar symbolizes quaternion conjugation, and $L_{1}=$ $u_{1}+i u_{2}+j u_{3}+k u_{4}, L_{2}=u_{5}+i u_{6}+j u_{7}+k u_{8}$, and $L_{3}=u_{9}$ are the quaternion and real control functions, respectively, that are to be determined. The quaternion systems (32) and (33) can be formed respectively as

$$
\left[\begin{array}{c}
\dot{x}_{d}(t) \\
\dot{y}_{d}(t) \\
\dot{z}_{d}(t)
\end{array}\right]=\left[\begin{array}{ccc}
-\alpha & \alpha & 0 \\
\gamma & -1 & 0 \\
0 & 0 & -\beta
\end{array}\right]\left[\begin{array}{c}
x_{d}(t) \\
y_{d}(t) \\
z_{d}(t)
\end{array}\right]+\left[\begin{array}{c}
0 \\
x_{d}(t) z_{d}(t) \\
\frac{1}{2}\left(y_{d}(t) \bar{x}_{d}(t)+x_{d}(t) \bar{y}_{d}(t)\right)
\end{array}\right]
$$

and

$$
\left[\begin{array}{c}
\dot{x}_{r}(t) \\
\dot{y}_{r}(t) \\
\dot{z}_{r}(t)
\end{array}\right]=\left[\begin{array}{ccc}
-\alpha & \alpha & 0 \\
\gamma & -1 & 0 \\
0 & 0 & -\beta
\end{array}\right]\left[\begin{array}{l}
x_{r}(t) \\
y_{r}(t) \\
z_{r}(t)
\end{array}\right]+\left[\begin{array}{c}
0 \\
x_{r}(t) z_{r}(t) \\
\frac{1}{2}\left(y_{r}(t) \bar{x}_{r}(t)+x_{r}(t) \bar{y}_{r}(t)\right)
\end{array}\right]+\left[\begin{array}{l}
L_{1} \\
L_{2} \\
L_{3}
\end{array}\right] .
$$

Thus, by comparing the quaternion systems (34) and (35) with the form of systems (21) and (22), respectively, we find that

$$
\begin{aligned}
\mathbf{A} & =\mathbf{B}=\left[\begin{array}{ccc}
-\alpha & \alpha & 0 \\
\gamma & -1 & 0 \\
0 & 0 & -\beta
\end{array}\right], \mathbf{f}\left(\mathbf{x}_{d}\right)=\left[\begin{array}{c}
0 \\
x_{d}(t) z_{d}(t) \\
0 \\
\frac{1}{2}\left(y_{d}(t) \bar{x}_{d}(t)+x_{d}(t) \bar{y}_{d}(t)\right)
\end{array}\right], \\
\mathbf{f}\left(\mathbf{x}_{r}\right) & =\left[\begin{array}{c}
x_{r}(t) z_{r}(t) \\
\frac{1}{2}\left(y_{r}(t) \bar{x}_{r}(t)+x_{r}(t) \bar{y}_{r}(t)\right.
\end{array}\right] .
\end{aligned}
$$

According to Theorem 1, the controller is designed as

$$
\mathbf{L}=-\mathbf{A} \mathbf{x}_{r}(t)-\mathbf{f}\left(\mathbf{x}_{r}(t)\right)-\mathbf{A} \mathbf{x}_{d}(t+\tau)-\mathbf{f}\left(\mathbf{x}_{d}(t+\tau)\right)-\zeta \mathbf{e}
$$


with

$$
\begin{aligned}
L_{1}= & -\alpha\left(y_{r}(t)-x_{r}(t)\right)-\alpha\left(y_{d}(t+\tau)-x_{d}(t+\tau)\right)-\zeta e_{1}, \\
u_{1}+i u_{2}+j u_{3}+k u_{4}= & -\alpha\left(e_{v_{5}}-e_{v_{1}}\right)-\zeta e_{v_{1}}-i\left[\alpha\left(e_{v_{6}}-e_{v_{2}}\right)-\zeta e_{v_{2}}\right] \\
& -j\left[\alpha\left(e_{v_{7}}-e_{v_{3}}\right)-\zeta e_{v_{3}}\right]-k\left[\alpha\left(e_{v_{8}}-e_{v_{4}}\right)-\zeta e_{v_{4}}\right], \\
L_{2}= & -\gamma x_{r}(t)+y_{r}(t)+\rho_{1}-\gamma x_{d}(t+\tau)-y_{d}(t+\tau)+\rho_{2}-\zeta e_{2}, \\
u_{5}+i u_{6}+j u_{7}+k u_{8}= & -\gamma e_{v_{1}}+e_{v_{5}}+\rho_{3}-\zeta e_{v_{5}}+i\left[-\gamma e_{v_{2}}+e_{v_{6}}+\rho_{4}-\zeta e_{v_{6}}\right] \\
& +j\left[-\gamma e_{v_{3}}+e_{v_{7}}+\rho_{5}-\zeta e_{v_{7}}\right]+k\left[-\gamma e_{v_{4}}+e_{v_{8}}+\rho_{6}-\zeta e_{v_{8}}\right], \\
L_{3}= & \beta z_{r}(t)-\rho_{7}+\beta z_{d}(t+\tau)-\rho_{8}-\zeta e_{3}, \\
u_{9}= & \beta e_{v_{9}}-\rho_{9}-\zeta e_{v_{9}},
\end{aligned}
$$

where $\rho_{1}=x_{r}(t) z_{r}(t), \rho_{2}=x_{d}(t+\tau) z_{d}(t+\tau), \rho_{3}=v_{1 r}(t) v_{9 r}(t)+v_{1 d}(t+\tau) v_{9 d}(t+\tau)$,

$$
\begin{aligned}
\rho_{4} & =v_{2 r}(t) v_{9 r}(t)+v_{2 d}(t+\tau) v_{9 d}(t+\tau), \rho_{5}=v_{3 r}(t) v_{9 r}(t)+v_{3 d}(t+\tau) v_{9 d}(t+\tau), \\
& \rho_{6}=v_{4 r}(t) v_{9 r}(t)+v_{4 d}(t+\tau) v_{9 d}(t+\tau), \rho_{7}=\frac{1}{2}\left(y_{r}(t) \bar{x}_{r}(t)+x_{r}(t) \bar{y}_{r}(t)\right), \\
\rho_{8} & =\frac{1}{2}\left(y_{d}(t+\tau) \bar{x}_{d}(t+\tau)+x_{d}(t+\tau) \bar{y}_{d}(t+\tau)\right), \\
\rho_{9} & =\left(v_{5 r}(t) v_{1 r}(t)+v_{5 d}(t+\tau) v_{1 d}(t+\tau)\right)+\left(v_{6 r}(t) v_{2 r}(t)+v_{6 d}(t+\tau) v_{2 d}(t+\tau)\right)+\left(v_{7 r}(t) v_{3 r}(t)+\right. \\
\left.v_{7 d}(t+\tau) v_{3 d}(t+\tau)\right) & \\
& +\left(v_{8 r}(t) v_{4 r}(t)+v_{8 d}(t+\tau) v_{4 d}(t+\tau)\right), \text { and } e_{v_{l}}=v_{l_{d}}(t+\tau)+v_{l r}(t)=0, l=1, \ldots, 9 .
\end{aligned}
$$

\subsection{Results of Numerical Analysis}

To validate the proposed approach, we shine the amusement results of the AAS between two similar chaotic quaternion structures (32) and (33) that, with the controller (36), are simulated numerically for $\alpha=25, \gamma=48$, and $\beta=5$. Our experiment was executed with the following initial conditions for the drive system: $\left[v_{1 d}(0)+i v_{2 d}(0)+\right.$ $\left.j v_{3 d}(0)+k v_{4 d}(0), v_{5 d}(0)+i v_{6 d}(0)+j v_{7 d}(0)+k v_{8 d}(0), v_{9 d}(0)\right]^{T}=[1+i 2+j 3+k 4,5+i 6+j 7+$ $k 8,9]^{T}$. For the response system, we select the initial conditions as $\left[v_{1 r}(0)+i v_{2 r}(0)+j v_{3 r}(0)+\right.$ $\left.k v_{4 r}(0), v_{5 r}(0)+i v_{6 r}(0)+j v_{7 r}(0)+k v_{8 r}(0), v_{9 r}(0)\right]^{T}=[1+i 2+j 3+k 4,5+i 6+j 7+k 8,9]^{T}$ with the positive anticipating time $\tau=0.2$. The results are shown in Figures 9-11. The results for the AAS of systems (32) and (33) are shown in Figure 9 for different initial conditions; these show that the AAS is actually achieved in a very short period of time $t$. Figure 10 shows the AAS errors; as predicted from the earlier analytical studies, the AAS errors $e_{v_{l}}$ decrease to zero as $t \rightarrow \infty$. As shown in Figure 11, the attractors in $\left(v_{9}, v_{1}, v_{3}\right)$ space (Figure 11a) and $\left(v_{9}, v_{8}, v_{2}\right)$ space (Figure 11b) of the drive structure (32) and response structure (33) have equal sizes but counter or opposite styles for anticipating time $\tau=0.2$. These result suggest that AAS is a reasonable type of synchronization for use in secure communications. This is because we were provided with a number of variables (quaternion numbers) that helped to secure the transfer of information. 
(a)

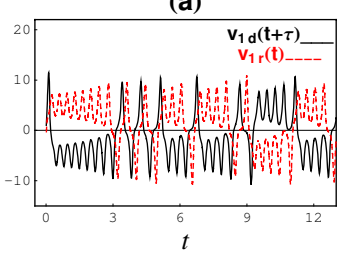

(d)

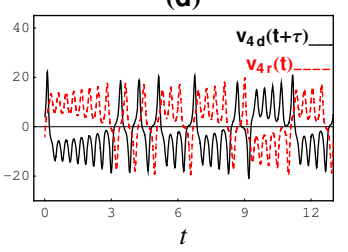

(g)

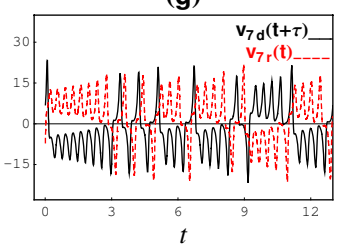

(b)

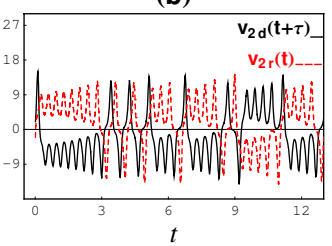

(e)

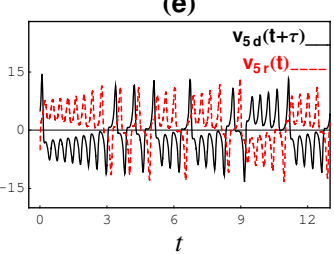

(h)

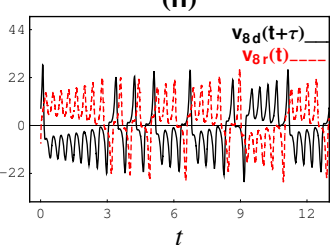

(c)

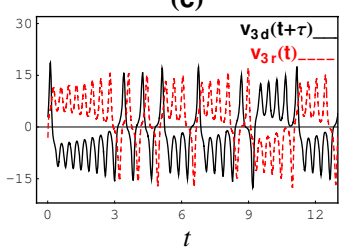

(f)

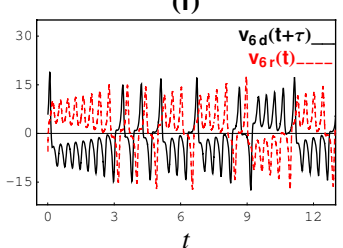

(i)

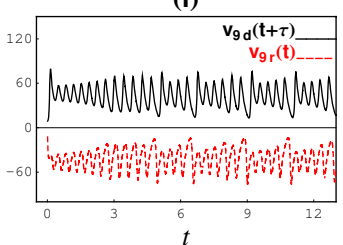

Figure 9. Numerical results of AAS of chaotic attractors of model (21) (solid curves) and model (22) (dashed curves). (a) $v_{1 d}(t+\tau)$ and $v_{1 r}(t)$ versus $t ;(\mathbf{b}) v_{2 d}(t+\tau)$ and $v_{2 r}(t)$ versus $t ;(\mathbf{c}) v_{3 d}(t+\tau)$ and $v_{3 r}(t)$ versus $t ;(\mathbf{d}) v_{4 d}(t+\tau)$ and $v_{4 r}(t)$ versus $t ;(\mathbf{e}) v_{5 d}(t+\tau)$ and $v_{5 r}(t)$ versus $t ;(\mathbf{f}) v_{6 d}(t+\tau)$ and $v_{6 r}(t)$ versus $t ;(\mathbf{g}) v_{7 d}(t+\tau)$ and $v_{7 r}(t)$ versus $t ;(\mathbf{h}) v_{8 d}(t+\tau)$ and $v_{8 r}(t)$ versus $t ;(\mathbf{i}) v_{9 d}(t+\tau)$ and $v_{9 r}(t)$ versus $t$.

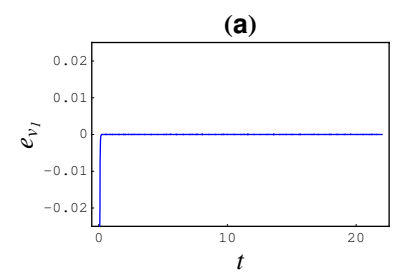

(d)

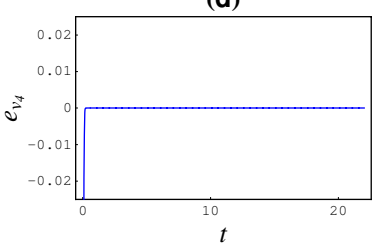

(g)

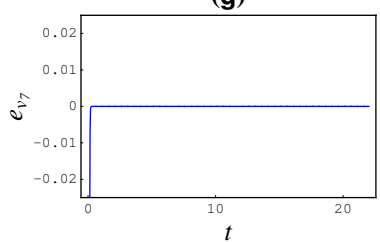

(b)

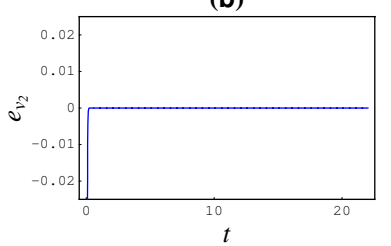

(e)

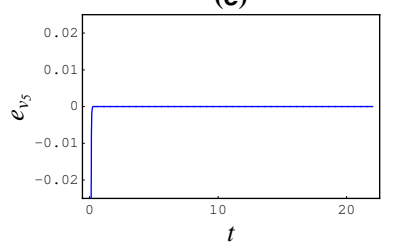

(h)

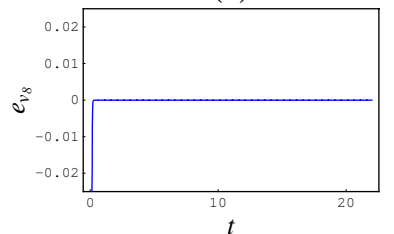

(c)

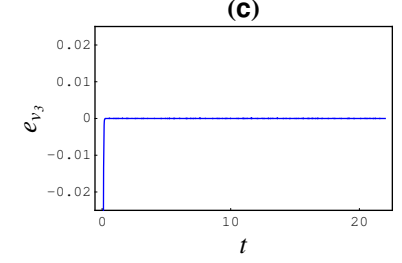

(f)

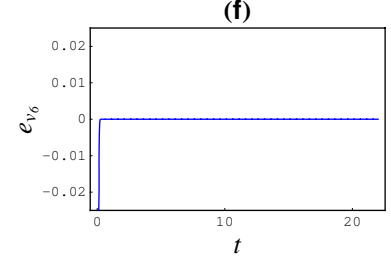

(i)

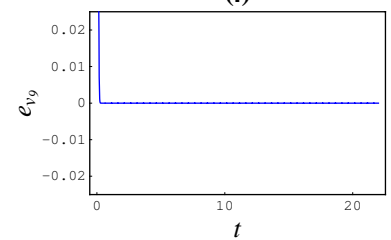

Figure 10. AAS errors (a) $\left(e_{v 1}, t\right)$ graph; (b) $\left(e_{v 2}, t\right)$ graph; $(\mathbf{c})\left(e_{v 3}, t\right)$ graph; $(\mathbf{d})\left(e_{v 4}, t\right)$ graph; (e) $\left(e_{v 5}, t\right)$ graph; $(\mathbf{f})\left(e_{v 6}, t\right)$ graph; $(\mathbf{g})\left(e_{v 7}, t\right)$ graph; $(\mathbf{h})\left(e_{v 8}, t\right)$ graph; $(\mathbf{i})\left(e_{v 9}, t\right)$ graph. 

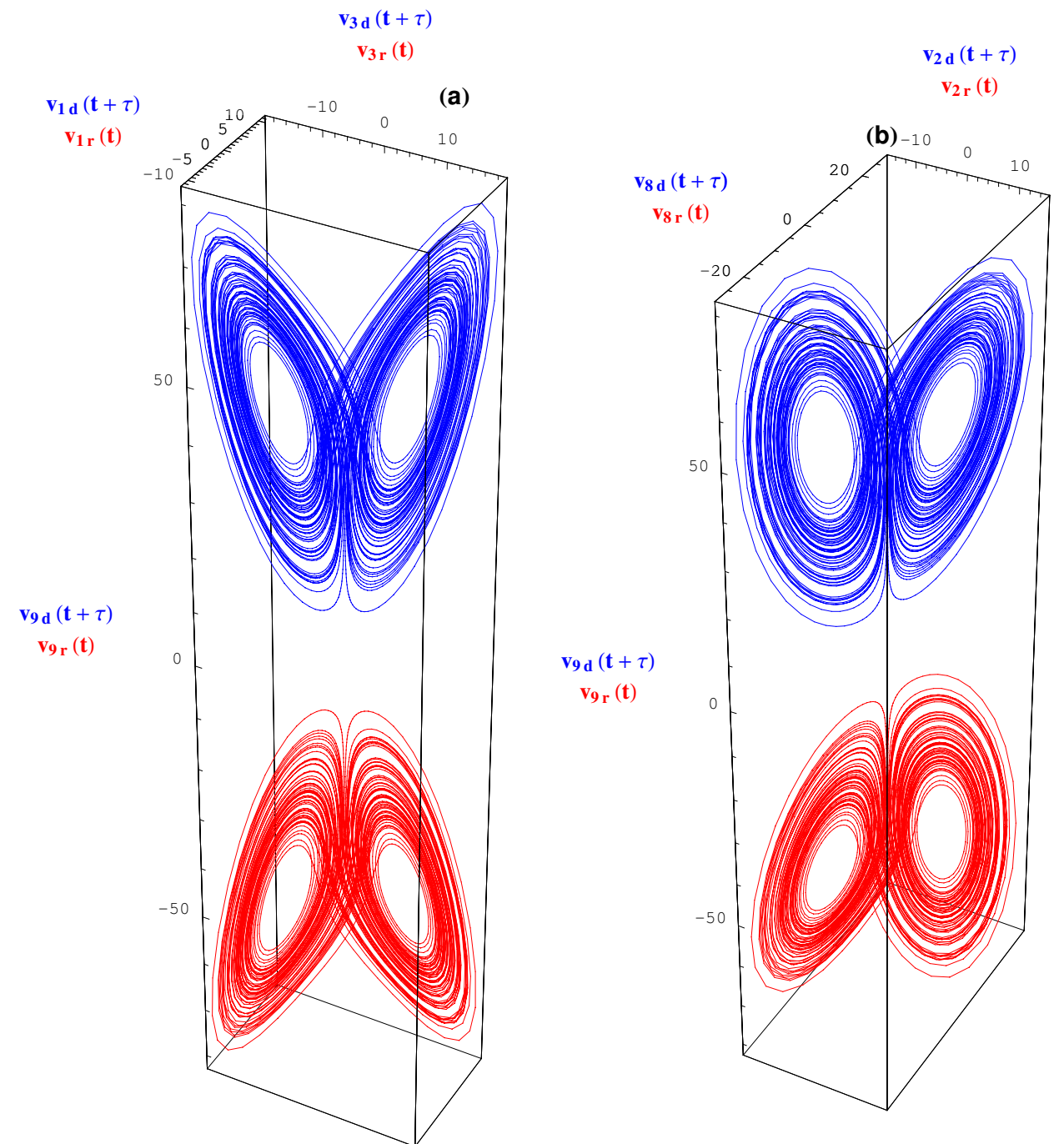

Figure 11. AAS of chaotic attractors of the drive system (21) (blue shape) and the response system (22) (red shape), (a) in $\left(v_{9}(t), v_{1}(t), v_{3}(t)\right)$ space; $(\mathbf{b})$ in $\left(v_{9}(t), v_{8}(t), v_{2}(t)\right)$ space.

\subsection{Secure Communication via AAS}

Chaotic secure communication has developed rapidly and performs a crucial role in the field of secure communication. Chaos theory is applied to chaotic communications with the intention of providing more security in the transmission of data via telecommunications technologies. In this work, we achieve chaotic communication security based on the chaotic Lorenz system with quaternion variables. The use of quadrilateral variables in secure communications is effective because of the increased number of dimensions, which helps to ensure exceptional security in data transmission. Unusual of the features of chaotic dynamics are used to encode data and mask, such as complex behavior, noise-like dynamics (pseudorandom noise), and spectral spread. Different communication techniques based on chaos have been offered [46-50]. The method of chaotic masking is used herein for the secure-communication application of our unusual chaotic system (4). In chaotic masking, the information (or message) signal $m(t)$ is combined with a chaotic driving signal, and the resulting modulated signal is transmitted to the receiver. At the receiver, AAS generates the same signal as the driving chaotic signal, thereby retrieving the same information signal as the original $m(t)$ information. To preserve AAS and prevent hostile attempts in the chaotic-masking method, in this research, we 
used new variables (quaternion numbers) and provided us with dynamics, which helps achieve safe communication.

The results of AAS of the new system were used to accomplish secure communications by utilizing an elementary structure (see Figure 12). The proposed structure has the drive system (21) as the transmitter system and the response system (22) as the receiver systems. The following invertible nonlinear function is used to encrypt the message signal $m(t)$ and chaotic signals of the transmitter:

$$
\begin{gathered}
\Xi=\phi\left(m(t), v_{1 d}(t+\tau), v_{2 d}(t+\tau), v_{3 d}(t+\tau), v_{4 d}(t+\tau), v_{5 d}(t+\tau),\right. \\
\left.v_{6 d}(t+\tau), v_{7 d}(t+\tau), v_{8 d}(t+\tau), v_{9 d}(t+\tau)\right) .
\end{gathered}
$$

We then add the signal $\Xi$ to one (or more) of the nine variables $v_{1 d}(t+\tau), v_{2 d}(t+\tau), v_{3 d}(t+\tau)$, $v_{4 d}(t+\tau), v_{5 d}(t+\tau), v_{6 d}(t+\tau), v_{7 d}(t+\tau), v_{8 d}(t+\tau)$, or $v_{9 d}(t+\tau)$; for instance, we could inject it into the variable $v_{9 d}(t+\tau)$ so that we get $\bar{m}(t)=\Xi+v_{9 d}(t+\tau)$ as the combined signal. Thus, the transmitter system sends its chaotic signals and the combined signal to the receiver side. In the receiver, the controller $\mathbf{L}$ can be constructed by Equation (37). Thus, the AAS between two identical chaotic quaternions (21), (22) systems the receiver starts to recover $\bar{m}(t)$ through a simple transformation $\Xi=\bar{m}(t)-v_{9 r}$. Finally, due to the invertability of the nonlinear function $\phi$, the message signal can be got back as $m^{*}(t)=\phi^{-1}\left(v_{1 r}(t), v_{2 r}(t), v_{3 r}(t), v_{4 r}(t), v_{5 r}(t), v_{6 r}(t), v_{7 r}(t), v_{8 r}(t), v_{9 r}(t), \Xi\right)$. We use the invertible function $\Xi=v_{1 d}(t+\tau)+m(t)$ with $m(t)=5 \sin 3 \pi t \cos 2 \pi t$, and we add the signal $\Xi$ to the variable $v_{9 d}(t+\tau)$ such that $\bar{m}(t)=v_{1 d}(t+\tau)+v_{9 d}(t+\tau)+m(t)$. A numerical simulation of using chaotic synchronization in secure communication is presented, in which the system parameters and the initial conditions of the transmitter and receiver systems are taken as those in Figure 9. Figure 13a,b show the message $m(t)$ and the transmitted signal $\bar{m}(t)$, respectively, Figure $13 \mathrm{c}$ shows the recovered message $m^{*}(t)=\bar{m}(t)-v_{1 r}(t)-v_{9 r}(t)$, and Figure 13d shows the information signal after filtering from error.

What distinguishes the results obtained when studying the security contacts in this work is the reliance on quaternion variables. Because using quaternion variables quadruples the transmitted contents and increases the diversity of synchronization, such use will vigorously enhance the security of communication. Therefore, secure communication based on quaternion variables has attracted intensive attention recently.

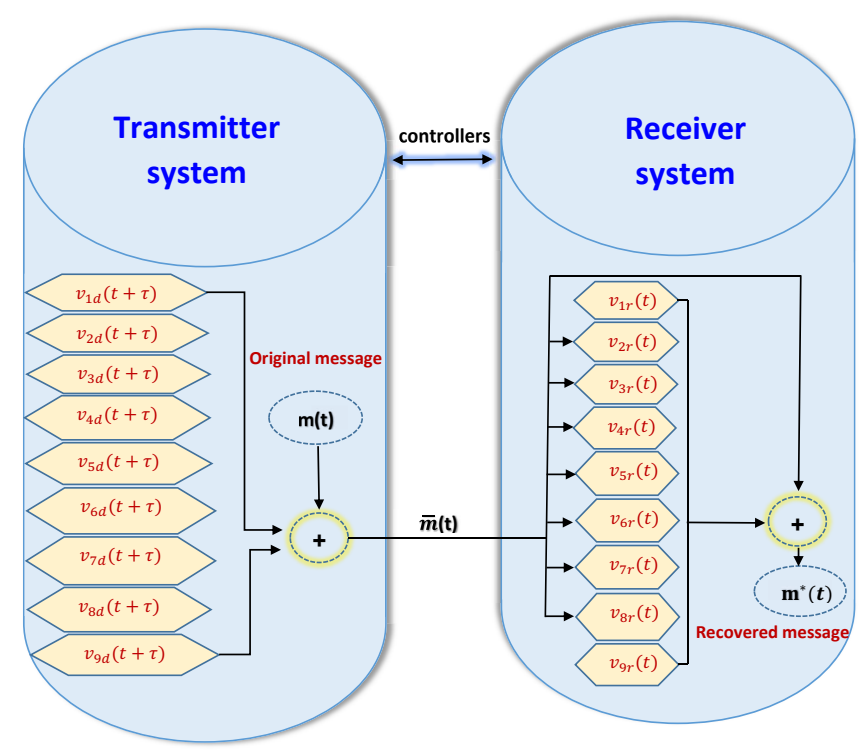

Figure 12. A scheme to realize secure communication between chaotic models (23) and (24) based on the results of AAS. 

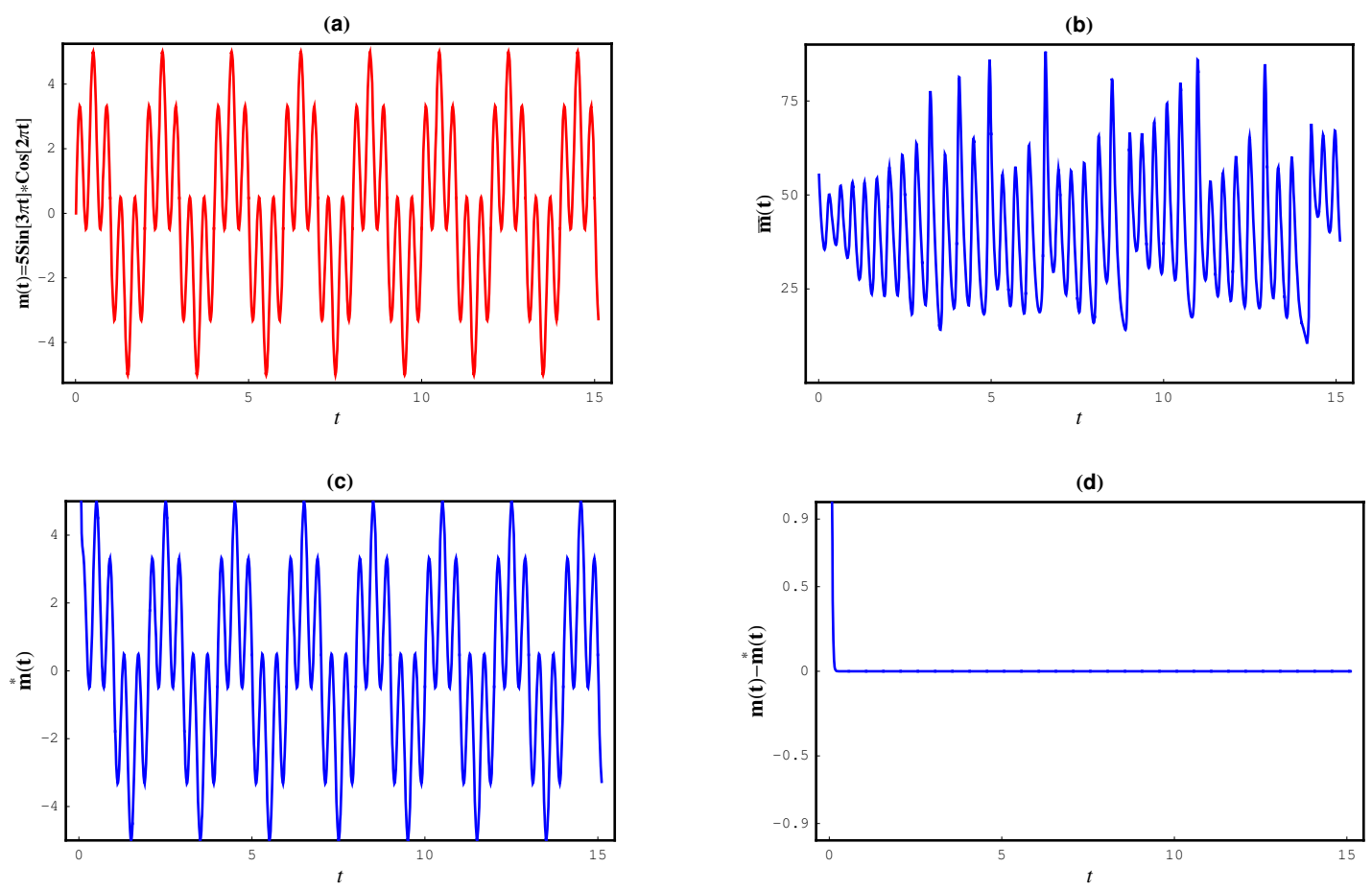

Figure 13. Results of secure communication. (a) the started message $m(t)$; (b) the transmitted signal $\bar{m}(t) ;(\mathbf{c})$ the recovered message $m^{*}(t) ;(\mathbf{d})$ the error signal $m(t)-m^{*}(t)$.

\section{Conclusions}

In recent years, several models of nonlinear dynamical systems have been presented with complex and real variables. However, to the best of our knowledge, quaternion variables had not been used to study nonlinear models. With quaternion variables, a high-dimension model is obtained if the real and imaginary parts are separated. Because of the need to increase the content and security of the transmitted information in communication, quadrupling the number of dimensions in our model is very helpful. Our high-dimension chaotic model is considered as a generalization of the real and complex Lorenz models. The proposed model (4) has nine real dimensions, and its complicated nonlinear response was studied. In addition, an electronic-circuit implementation of system (4) was designed and constructed as a Simulink simulation, and this gave good agreement with the analytical results.

We proposed a new technique to realize AAS between a pair of similar chaotic quaternion nonlinear models. To realize AAS, the quaternion controller is regulated analytically so that, in the AAS, the response dynamics act as a predictor of the drive dynamics with the opposite sign. We provided an example to illustrate our proposed technique, wherein the results of AAS were applied to give a simple implementation in safe communications. The use of complicated chaotic schemes in communications motivated us to study the basics of data transmission. In this study, we have proposed an extension of secure communication methods by using not only real and complex signals, but also quaternion chaotic models. The dynamics of quaternion dynamical models have been made clearer by the present detailed results.

Author Contributions: Conceptualization, E.E.M., M.H. and T.M.A.-H.; methodology, E.E.M., M.H. and T.M.A.-H.; software, E.E.M., M.H. and T.M.A.-H.; validation, E.E.M., M.H. and T.M.A.-H.; formal analysis, E.E.M., M.H. and T.M.A.-H.; investigation, E.E.M., M.H. and T.M.A.-H.; resources, E.E.M., M.H. and T.M.A.-H.; data curation, E.E.M., M.H. and T.M.A.-H.; writing-original draft preparation, E.E.M., M.H. and T.M.A.-H.; writing-review and editing, E.E.M., M.H. and T.M.A.-H.; visualization, E.E.M. and M.H.; supervision, E.E.M.

Funding: This research received no external funding.

Conflicts of Interest: The authors declare no conflict of interest. 


\section{References}

1. Lam, T.Y. Hamilton's quaternions. Handb. Algebra 2003, 3, 429-454.

2. Al-Zhour, Z. Some new linear representations of matrix quaternions with some applications. J. King Saud Univ. Sci. 2017, 31, 42-47. [CrossRef]

3. Vaidyanathan, S. Analysis adaptive control and synchronization of a novel 4-D hyperchaotic hyperjerk system via backstepping control method. Arch. Control Sci. 2016, 26, 311-338. [CrossRef]

4. Voss, H.U. Anticipating chaotic synchronization. Phys. Rev. 2000, 61, 5115. [CrossRef] [PubMed]

5. Voss, H.U. Dynamic long-term anticipation of chaotic states. Phys. Rev. Lett. 2001, 87, 014102. [CrossRef] [PubMed]

6. Masoller, A.P.C. Anticipation in the synchronization of chaotic semiconductor lasers with optical feedback. Phys. Rev. Lett. 2001, 86, 2782. [CrossRef] [PubMed]

7. Pyragienè, T.; Pyragas, K. Anticipating synchronization in a chain of chaotic oscillators with switching parameters. Phys. Lett. 2015, 379, 3084-3088. [CrossRef]

8. Mousa, S.K.; Al Naimee, K.A. Experimental investigations of Synchronization in two optically coupled chaotic systems utilizing optical feedback and optical injection. J. Univ. Anbar Pure Sci. 2017, 11, 49-56.

9. Pavlos, G.P.; Karakatsanis, L.P.; Xenakis, M.N. Tsallis non-extensive statistics intermittent turbulence SOC and chaos in the solar plasma Part one Sunspot dynamics. Phys. Stat. Mech. Appl. 2012, 391, 6287-6319. [CrossRef]

10. Roy, A.; Misra, A.P.; Banerjee, S. Chaos-based image encryption using vertical-cavity surface-emitting lasers. Optik 2019, 176, 119-131. [CrossRef]

11. Fen, M.O. Persistence of chaos in coupled Lorenz systems Chaos. Solitons Fractals 2017, 95, $200-205$. [CrossRef]

12. Li, J.; Lu, M.; Zhang, W.; Qian, Y. A new butterfly attractor based on Chua's circuit and its application in security communication. In Proceedings of the 2013 IEEE International Conference of IEEE Region 10 (TENCON 2013), Xi'an, China, 22-25 October 2013; Volume 10, pp. 1-4.

13. Sprott, J.C.; Chen, G. Coexistence of point periodic and strange attractors. Int. J. Bifurc. Chaos 2013, 23, 1350093. [CrossRef]

14. Boeing, G. Visual Analysis of Nonlinear Dynamical Systems: Chaos, Fractals, Self-Similarity and the Limits of Prediction. Systems 2016, 4, 37. [CrossRef]

15. Strogatz, S.; Friedman, M.; Mallinckrodt, A.J.; McKay, S. Nonlinear dynamics and chaos with applications to physics biology chemistry and engineering. Comput. Phys. 1994, 8, 532. [CrossRef]

16. Wahab, H.B.A.; Abed, T.M. E-commerce Application Based on Visual Cryptography and Chen's Hyperchaotic. Iraqi J. Sci. 2018, 59, 617-628.

17. Pham, V.T.; Volos, C.K. Advances and Applications in Chaotic Systems; Springer: Berlin, Germany, 2016; Volume 636.

18. Sparrow, C. The Lorenz Equations Bifurcations Chaos and Strange Attractors; Springer: Berlin, Germany, 2012; Volume 41.

19. Mahmoud, E.E. Dynamics and synchronization of new hyperchaotic complex Lorenz system. Math. Comput. Model. 2012, 55, 951-1962. [CrossRef]

20. Pehlivan, I.; Yilmaz, U. A new chaotic attractor from general Lorenz system family and its electronic experimental implementation. Turk. J. Electr. Eng. Comput. Sci. 2010, 18, 171-184.

21. Mahmoud, E.E.; Al-Adwani, M.A. Dynamical behaviors, control and synchronization of a new chaotic model with complex variables and cubic nonlinear terms. Results Phys. 2017, 7, 1346-1356. [CrossRef]

22. Korenberg, M.J.; Hunter, I.W. The identification of nonlinear biological systems: Volterra kernel approaches. Ann. Biomed. Eng. 1996, 24, 250-268. [CrossRef]

23. Mahmoud, G.M.; Mahmoud, E.E. Complex modified projective synchronization of two chaotic complex nonlinear systems. Nonlinear Dyn. 2013, 73, 2231-2240. [CrossRef]

24. Mahmoud, E.E.; Abood, F.S. A new nonlinear chaotic complex model and its complex antilag synchronization. Complexity 2017, 2017, 13p. [CrossRef] 
25. Mahmoud, E.E.; Abo-Dahab, S.M. Dynamical properties and complex anti synchronization with applications to secure communications for a novel chaotic complex nonlinear model. Solitons Fractals 2018, 106, 273-284. [CrossRef]

26. Mahmoud, E.E. Complex complete synchronization of two nonidentical hyperchaotic complex nonlinear systems. Math. Methods Appl. Sci. 2014, 37, 321-328. [CrossRef]

27. Hu, J.; Chunna, Z.; Tan, J. Boundedness and periodicity for linear threshold discrete-time quaternion-valued neural network with time-delays. Neurocomputing 2017, 267, 417-425. [CrossRef]

28. Finkelstein, D.; Jauch, J.M.; Schiminovich, S.; Speiser, D. Foundations of quaternion quantum mechanics. J. Math. Phys. 1962, 3, 207-220. [CrossRef]

29. Catoni, F.; Cannata, R.; Zampetti, P. An introduction to commutative quaternions. Adv. Appl. Clifford Algebr. 2006, 16, 1-28. [CrossRef]

30. Tweed, D.; Douglas, W.; Vilis, T. Computing three-dimensional eye position quaternions and eye velocity from search coil signals. Vis. Res. 1990, 30, 97-110. [CrossRef]

31. Song, G.J.; Wang, Q.W.; Yu, S.W. Cramer's rule for a system of quaternion matrix equations with applications. Appl. Math. Comput. 2018, 336, 490-499. [CrossRef]

32. Frenkel, I.; Libine, M. Quaternionic analysis representation theory and physics. Adv. Math. 2008, 218, 1806-1877. [CrossRef]

33. Girard, P.R. The quaternion group and modern physics. Eur. J. Phys. 1984, 5, 25. [CrossRef]

34. Aydın, F.T. Bicomplex Fibonacci quaternions Chaos. Solitons Fractals 2018, 106, 147-153. [CrossRef]

35. Quezada-Téllez, L.A.; Carrillo-Moreno, S.; Rosas-Jaimes, O.; Flores-Godoy, J.J.; Fernndez-Anaya, G. Dynamic analysis of a Lü model in six dimensions and its projections. Int. J. Nonlinear Sci. Numer. Simul. 2017, 18, 371-384. [CrossRef]

36. Schmid, H.; Huber, A. Analysis of switched-capacitor circuits using driving-point signal-flow graphs. Analog Integr. Circuits Signal Process 2018, 96, 495-507. [CrossRef]

37. Smith, P.; Jordan, D.W. Nonlinear Ordinary Differential Equations Problems and Solutions; Oxford University Press: Oxford, UK, 2007; pp. 523-528.

38. $\mathrm{Hu}, \mathrm{G}$. Generating hyperchaotic attractors with three positive lyapunov exponents via state feedback control. Int. J. Bifurc. Chaos 2009, 19, 651-660. [CrossRef]

39. Wolf, A.; Swift, J.B.; Swinney, H.L.; Vastano, J.A. Determining Lyapunov exponents from a time series. Phys. D Nonlinear Phenom. 1985, 16, 285-317. [CrossRef]

40. Frederickson, P.; Kaplan, J.L.; Yorke, E.D.; Yorke, J.A. The Liapunov dimension of strange attractors. J. Differ. Equ. 1983, 49, 185-207. [CrossRef]

41. Mahmoud, E.E.; AL-Harthi, B.H. A phenomenal form of complex synchronization and chaotic masking communication between two identical chaotic complex nonlinear structures with unknown parameters. Results Phys. 2019, 14, 102452. [CrossRef]

42. Huang, C.; Cao, J. Active control strategy for synchronization and anti-synchronization of a fractional chaotic financial system. Stat. Mech. Appl. 2017, 473, 262-275. [CrossRef]

43. Ma, J.; Li, F.; Huang, L.; Jin, W.Y. Complete synchronization phase synchronization and parameters estimation in a realistic chaotic system. Commun. Nonlinear Sci. Numer. Simul. 2011, 16, 3770-3785. [CrossRef]

44. Zhang, W.; Cao, J.; Wu, R.; Alsaadi, F.E.; Alsaedi, A. Lag projective synchronization of fractional-order delayed chaotic systems. J. Frankl. Inst. 2019, 356, 1522-1534. [CrossRef]

45. Huang, T.; Gao, D.; Li, C.; Xiao, M.Q. Anticipating synchronization through optimal feedback control. J. Glob. Optim. 2012, 52, 281-290. [CrossRef]

46. Mahmoud, E.E.; Abualnaja, K.M.; Althagafi, O.A. High dimensional, four positive Lyapunov exponents and attractors with four scroll during a new hyperchaotic complex nonlinear model. AIP Adv. 2018, 8, 065018. [CrossRef]

47. Mahmoud, E.E.; AL-Harthi, B.H. Secure communications via modified complex phase synchronization of two hyperchaotic complex models with identical linear structure and adjusting in nonlinear terms. J. Intell. Fuzzy Syst. 2019, 37, 17-25. [CrossRef]

48. Mahmoud, G.M.; Mahmoud, E.E.; Arafa, A.A. Projective synchronization for coupled partially linear complex-variable systems with known parameters. Math. Methods Appl. Sci. 2017, 40, 1214-1222. [CrossRef] 
49. Sahina, M.E.; Camh, Z.G.; Gulerce, H.; Hamamci, S.E. Simulation and Circuit Implementation Memristive Chaotic System and Its Application for Secure Communication Systems. Sens. Actuators Phys. 2019, 290, 107-118. [CrossRef]

50. Ekhande, R.; Deshmukh, S. Chaotic Signal for Signal Masking in Digital Communications. Int. Org. Sci. Res. J. Eng. 2014, 4, 29-33. [CrossRef]

(C) 2019 by the authors. Licensee MDPI, Basel, Switzerland. This article is an open access article distributed under the terms and conditions of the Creative Commons Attribution (CC BY) license (http:/ / creativecommons.org/licenses/by/4.0/). 